\title{
SYMMETRIC PROPERTIES OF REAL FUNCTIONS
}




\title{
PURE AND APPLIED MATHEMATICS
}

\section{A Program of Monographs, Textbooks, and Lecture Notes}

\section{EXECUTIVE EDITORS}

\author{
Earl J. Taft \\ Rutgers University \\ New Brunswick, New Jersey
}

\author{
Zuhair Nashed \\ University of Delaware \\ Newark, Delaware
}

\section{CHAIRMEN OF THE EDITORIAL BOARD}

\author{
S. Kobayashi \\ University of California, Berkeley \\ Berkeley, California
}

\author{
Edwin Hewitt \\ University of Washington \\ Seattle, Washington
}

\section{EDITORIAL BOARD}

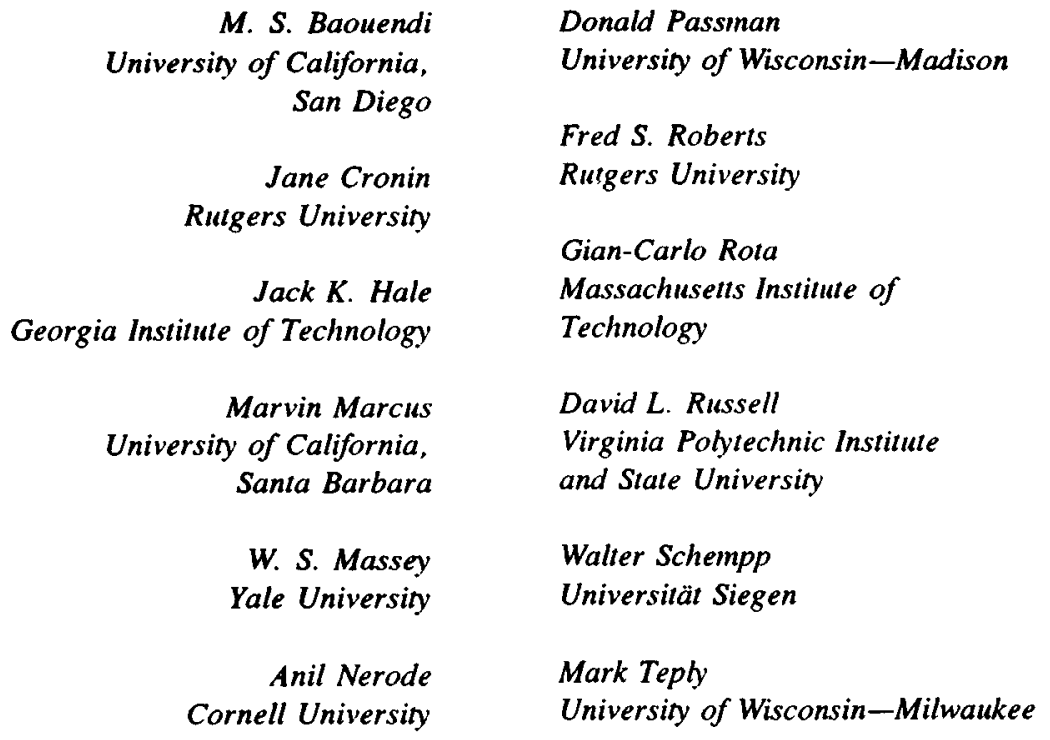




\section{MONOGRAPHS AND TEXTBOOKS IN PURE AND APPLIED MATHEMATICS}

1. K. Yano, Integral Formulas in Riemannian Geometry (1970)

2. S. Kobayashi, Hyperbolic Manifolds and Holomorphic Mappings (1970)

3. V. S. Vladimirov, Equations of Mathematical Physics (A. Jeffrey, ed.; A. Littlewood, trans.) (1970)

4. B. N. Pshenichnyi, Necessary Conditions for an Extremum (L. Neustadt, translation ed.; K. Makowski, trans.) (1971)

5. L. Narici ot al., Functional Analysis and Valuation Theory (1971)

6. S. S. Passman, Infinite Group Rings (1971)

7. L. Dornhoff, Group Representation Theory. Part A: Ordinary Representation Theory. Part B: Modular Representation Theory $(1971,1972)$

8. W. Boothby and G. L. Weiss, eds., Symmetric Spaces (1972)

9. Y. Matsushima, Differentiable Manifolds (E. T. Kobayashi, trans.) (1972)

10. L. E. Ward, Jr., Topology (1972)

11. A. Babakhanian, Cohomological Methods in Group Theory (1972)

12. R. Gilmer, Multiplicative Ideal Theory (1972)

13. J. Yeh, Stochastic Processes and the Wiener Integral (1973)

14. J. Barros-Neto, Introduction to the Theory of Distributions (1973)

15. R. Larsen, Functional Analysis (1973)

16. K. Yano and S. Ishihara, Tangent and Cotangent Bundles (1973)

17. C. Procesi, Rings with Polynomial Identities (1973)

18. R. Hermann, Geometry, Physics, and Systems (1973)

19. N. R. Wallach, Harmonic Analysis on Homogeneous Spaces (1973)

20. J. Dieudonné, Introduction to the Theory of Formal Groups (1973)

21. I. Vaisman, Cohomology and Differential Forms (1973)

22. B. -Y. Chen, Geometry of Submanifolds (1973)

23. M. Marcus, Finite Dimensional Multilinear Algebra (in two parts) $(1973,1975)$

24. R. Larsen, Banach Algebras (1973)

25. R. O. Kujala and A. L. Vitter, eds., Value Distribution Theory: Part A; Part B: Deficit and Bezout Estimates by Wilhelm Stoll (1973)

26. K. B. Stolarsky, Algebraic Numbers and Diophantine Approximation (1974)

27. A. R. Magid, The Separable Galois Theory of Commutative Rings (1974)

28. B. R. McDonald, Finite Rings with Identity (1974)

29. J. Satake, Linear Algebra (S. Koh et al., trans.) (1975)

30. J. S. Golan, Localization of Noncommutative Rings (1975)

31. G. Klambauer, Mathematical Analysis (1975)

32. M. K. Agoston, Algebraic Topology (1976)

33. K. R. Goodearl, Ring Theory (1976)

34. L. E. Mansfield, Linear Algebra with Geometric Applications (1976)

35. N. J. Pullman, Matrix Theory and Its Applications (1976)

36. B. R. McDonald, Geometric Algebra Over Local Rings (1976)

37. C. W. Groetsch, Generalized Inverses of Linear Operators (1977)

38. J. E. Kuczkowski and J. L. Gersting, Abstract Algebra (1977)

39. C. O. Christenson and W. L. Voxman, Aspects of Topology (1977)

40. M. Nagata, Field Theory (1977)

41. R. L. Long, Algebraic Number Theory (1977)

42. W. F. Pfeffer, Integrals and Measures (1977)

43. R. L. Wheeden and A. Zygmund, Measure and Integral (1977)

44. J. H. Curtiss, Introduction to Functions of a Complex Variable (1978)

45. K. Hrbacek and T. Jech, Introduction to Set Theory (1978)

46. W. S. Massey, Homology and Cohomology Theory (1978)

47. M. Marcus, Introduction to Modern Algebra (1978)

48. E. C. Young, Vector and Tensor Analysis (1978)

49. S. B. Nadler, Jr., Hyperspaces of Sets (1978)

50. S. K. Segal, Topics in Group Kings (1978)

51. A. C. M. van Rooij, Non-Archimedean Functional Analysis (1978)

52. L. Corwin and R. Szczarba, Calculus in Vector Spaces (1979) 
53. C. Sadosky, Interpolation of Operators and Singular Integrals (1979)

54. J. Cronin, Differential Equations (1980)

55. C. W. Groetsch, Elements of Applicable Functional Analysis (1980)

56. I. Vaisman, Foundations of Three-Dimensional Euclidean Geometry (1980)

57. H. I. Freeden, Deterministic Mathematical Models in Population Ecology (1980)

58. S. B. Chae, Lebesgue Integration (1980)

59. C. S. Rees et al., Theory and Applications of Fourier Analysis (1981)

60. L. Nachbin, Introduction to Functional Analysis (R. M. Aron, trans.) (1981)

61. G. Orzech and M. Orzech, Plane Algebraic Curves (1981)

62. R. Johnsonbaugh and W. E. Pfaffenberger, Foundations of Mathematical Analysis (1981)

63. W. L. Voxman and R. H. Goetschel, Advanced Calculus (1981)

64. L. J. Corwin and R. H. Szcarba, Multivariable Calculus (1982)

65. V. I. Istrătescu, Introduction to Linear Operator Theory (1981)

66. R. D. Järvinen, Finite and Infinite Dimensional Linear Spaces (1981)

67. J. K. Beem and P. E. Ehrlich, Global Lorentzian Geometry (1981)

68. D. L. Armacost, The Structure of Locally Compact Abelian Groups (1981)

69. J. W. Brewer and M. K. Smith, eds., Emily Noether: A Tribute (1981)

70. K. H. Kim, Boolean Matrix Theory and Applications (1982)

71. T. W. Wieting, The Mathematical Theory of Chromatic Plane Ornaments (1982)

72. D. B. Gauld, Differential Topology (1982)

73. R. L. Faber, Foundations of Euclidean and Non-Euclidean Geometry (1983)

74. M. Carmeli, Statistical Theory and Random Matrices (1983)

75. J. H. Carruth et al., The Theory of Topological Semigroups (1983)

76. R. L. Faber, Differential Geometry and Relativity Theory (1983)

77. S. Barnett, Polynomials and Linear Control Systems (1983)

78. G. Karpilovsky, Commutative Group Algebras (1983)

79. F. Van Oystaeyen and A. Verschoren, Relative Invariants of Rings (1983)

80. 1. Vaisman, A First Course in Differential Geometry (1984)

81. G. W. Swan, Applications of Optimal Control Theory in Biomedicine (1984)

82. T. Petrie and J. D. Randall, Transformation Groups on Manifolds (1984)

83. K. Goebel and S. Reich, Uniform Convexity, Hyperbolic Geometry, and Nonexpansive Mappings (1984)

84. T. Albu and C. Năstăsescu, Relative Finiteness in Module Theory (1984)

85. K. Hrbacek and T. Jech, introduction to Set Theory: Second Edition (1984)

86. F. Van Oystaeyen and A. Verschoren, Relative Invariants of Rings (1984)

87. B. R. McDonald, Linear Algebra Over Commutative Rings (1984)

88. M. Namba, Geometry of Projective Algebraic Curves (1984)

89. G. F. Webb, Theory of Nonlinear Age-Dependent Population Dynamics (1985)

90. M. R. Bremner et al., Tables of Dominant Weight Multiplicities for Representations of Simple Lie Algebras (1985)

91. A. E. Fekete, Real Linear Algebra (1985)

92. S. B. Chae, Holomorphy and Calculus in Normed Spaces (1985)

93. A. J. Jerri, Introduction to Integral Equations with Applications (1985)

94. G. Karpilovsky, Projective Representations of Finite Groups (1985)

95. L. Narici and E. Beckenstein, Topological Vector Spaces (1985)

96. J. Weeks, The Shape of Space (1985)

97. P. R. Gribik and K. O. Kortanek, Extremal Methods of Operations Research (1985)

98. J.-A. Chao and W. A. Woyczynski, eds., Probability Theory and Harmonic Analysis (1986)

99. G. D. Crown et al., Abstract Algebra (1986)

100. J. H. Carruth et al., The Theory of Topological Semigroups, Volume 2 (1986)

101. R. S. Doran and V. A. Belfi, Characterizations of $C^{*}$-Algebras (1986)

102. M. W. Jeter, Mathematical Programming (1986)

103. M. Altman, A Unified Theory of Nonlinear Operator and Evolution Equations with Applications (1986)

104. A. Verschoren, Relative Invariants of Sheaves (1987)

105. R. A. Usmani, Applied Linear Algebra (1987)

106. P. Blass and J. Lang, Zariski Surfaces and Differential Equations in Characteristic $p>$ 0 (1987)

107. J. A. Reneke et al., Structured Hereditary Systems (1987) 
108. H. Busemann and B. B. Phadke, Spaces with Distinguished Geodesics (1987)

109. R. Harte, Invertibility and Singularity for Bounded Linear Operators (1988)

110. G. S. Ladde et al., Oscillation Theory of Differential Equations with Deviating Arguments (1987)

111. L. Dudkin et al., Iterative Aggregation Theory (1987)

112. T. Okubo, Differential Geometry (1987)

113. D. L. Stancl and M. L. Stancl, Real Analysis with Point-Set Topology (1987)

114. T. C. Gerd, Introduction to Stochastic Differential Equations (1988)

115. S. S. Abhyankar, Enumerative Combinatorics of Young Tableaux (1988)

116. H. Strade and R. Farnsteiner, Modular Lie Algebras and Their Representations (1988)

117. J. A. Huckaba, Commutative Rings with Zero Divisors (1988)

118. W. D. Wallis, Combinatorial Designs (1988)

119. W. Więstaw, Topological Fields (1988)

120. G. Karpilovsky, Field Theory (1988)

121. S. Caenepeel and F. Van Oystaeyen, Brauer Groups and the Cohomology of Graded Rings (1989)

122. W. Kozlowski, Modular Function Spaces (1988)

123. E. Lowen-Colebunders, Function Classes of Cauchy Continuous Maps (1989)

124. M. Pavel, Fundamentals of Pattern Recognition (1989)

125. V. Lakshmikantham et al., Stability Analysis of Nonlinear Systems (1989)

126. R. Sivaramakrishnan, The Classical Theory of Arithmetic Functions (1989)

127. N. A. Watson, Parabolic Equations on an Infinite Strip (1989)

128. K. J. Hastings, Introduction to the Mathematics of Operations Research (1989)

129. B. Fine, Algebraic Theory of the Bianchi Groups (1989)

130. D. N. Dikranjan ot al., Topological Groups (1989)

131. J. C. Morgan II, Point Set Theory (1990)

132. P. Biler and A. Witkowski, Problems in Mathematical Analysis (1990)

133. H. J. Sussmann, Nonlinear Controllability and Optimal Control (1990)

134. J.-P. Florens et al., Elements of Bayesian Statistics (1990)

135. N. Shell, Topological Fields and Near Valuations (1990)

136. B. F. Doolin and C. F. Martin, Introduction to Differential Geometry for Engineers (1990)

137. S. S. Holland, Jr., Applied Analysis by the Hilbert Space Method (1990)

138. J. Okniński, Semigroup Algebras (1990)

139. $K$. Zhu, Operator Theory in Function Spaces (1990)

140. G. B. Price, An Introduction to Multicomplex Spaces and Functions (1991)

141. R. B. Darst, Introduction to Linear Programming (1991)

142. P. L. Sachdev, Nonlinear Ordinary Differential Equations and Their Applications (1991)

143. T. Husain, Orthogonal Schauder Bases (1991)

144. J. Foran, Fundamentals of Real Analysis (1991)

145. W. C. Brown, Matrices and Vector Spaces (1991)

146. M. M. Rao and Z. D. Ren, Theory of Orlicz Spaces (1991)

147. J. S. Golan and T. Head, Modules and the Structures of Rings (1991)

148. C. Small, Arithmetic of Finite Fields (1991)

149. K. Yang, Complex Algebraic Geometry (1991)

150. D. G. Hoffman et al., Coding Theory (1991)

151. M. O. González, Classical Complex Analysis (1992)

152. M. O. González, Complex Analysis (1992)

153. L. W. Baggett, Functional Analysis (1992)

154. M. Sniedovich, Dynamic Programming (1992)

155. R. P. Agarwal, Difference Equations and Inequalities (1992)

156. C. Brezinski, Biorthogonality and Its Applications to Numerical Analysis (1992)

157. C. Swartz, An Introduction to Functional Analysis (1992)

158. S. B. Nadler, Jr., Continuum Theory (1992)

159. M. A. Al-Gwaiz, Theory of Distributions (1992)

160. E. Perry, Geometry: Axiomatic Developments with Problem Solving (1992)

161. E. Castillo and M. R. Ruiz-Cobo, Functional Equations and Modelling in Science and Engineering (1992)

162. A. J. Jerri, Integral and Discrete Transforms with Applications and Error Analysis (1992)

163. A. Charlier et al., Tensors and the Clifford Algebra (1992) 
164. P. Biler and T. Nadzieja, Problems and Examples in Differential Equations (1992)

165. E. Hansen, Global Optimization Using Interval Analysis (1992)

166. S. Guerre-Delabrière, Classical Sequences in Banach Spaces (1992)

167. Y. C. Wong, Introductory Theory of Topological Vector Spaces (1992)

168. S. H. Kulkarni and B. V. Limaye, Real Function Algebras (1992)

169. W. C. Brown, Matrices Over Commutative Rings (1993)

170. J. Loustau and M. Dillon, Linear Geometry with Computer Graphics (1993)

171. W. V. Petryshyn, Approximation-Solvability of Nonlinear Functional and Differential Equations (1993)

172. E. C. Young, Vector and Tensor Analysis: Second Edition (1993)

173. T. A. Bick. Elementary Boundary Value Problems (1993)

174. M. Pavel, Fundamentals of Pattern Recognition: Second Edition (1993)

175. S. A. Albeverio et al., Noncommutative Distributions (1993)

176. W. Fulks, Complex Variables (1993)

177. M. M. Rao, Conditional Measures and Applications (1993)

178. A. Janicki and A. Weron, Simulation and Chaotic Behavior of $a$-Stable Stochastic Processes (1994)

179. P. Neittaanmäki and D. Tiba, Optimal Control of Nonlinear Parabolic Systems (1994)

180. J. Cronin, Differential Equations: Introduction and Qualitative Theory, Second Edition (1994)

181. S. Heikkilä and V. Lakshmikantham, Montotone Iterative Techniques for Discontinuous Nonlinear Differential Equations (1994)

182. X. Mao. Exponential Stability of Stochastic Differential Equations (1994)

183. B. S. Thomson, Symmetric Properties of Real Functions (1994) 


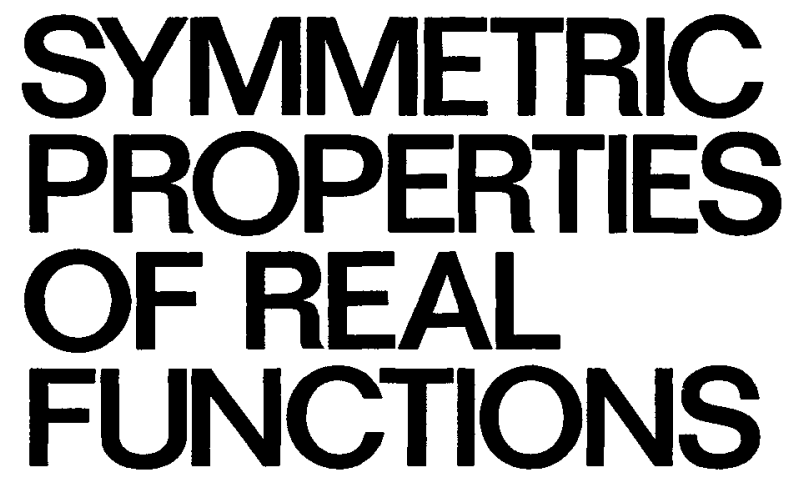

\section{Brian S. Thomson}

\section{Simon Fraser University}

British Columbia, Canada 
CRC Press

Taylor \& Francis Group

6000 Broken Sound Parkway NW, Suite 300

Boca Raton, FL 33487-2742

First issued in paperback 2019

(C) 1994 by Taylor \& Francis Group, LLC

CRC Press is an imprint of Taylor \& Francis Group, an Informa business

No claim to original U.S. Government works

ISBN-13: 978-0-8247-9230-5 (hbk)

ISBN-13: 978-0-367-40203-7 (pbk)

This book contains information obtained from authentic and highly regarded sources. Reasonable efforts have been made to publish reliable data and information, but the author and publisher cannot assume responsibility for the validity of all materials or the consequences of their use. The authors and publishers have attempted to trace the copyright holders of all material reproduced in this publication and apologize to copyright holders if permission to publish in this form has not been obtained. If any copyright material has not been acknowledged please write and let us know so we may rectify in any future reprint.

Except as permitted under U.S. Copyright Law, no part of this book may be reprinted, reproduced, transmitted, or utilized in any form by any electronic, mechanical, or other means, now known or hereafter invented, including photocopying, microfilming, and recording, or in any information storage or retrieval system, without written permission from the publishers

For permission to photocopy or use material electronically from this work, please access www. copyright.com (http://www.copyright.com/) or contact the Copyright Clearance Center, Inc. (CCC), 222 Rosewood Drive, Danvers, MA 01923, 978-750-8400. CCC is a not-for-profit organization that provides licenses and registration for a variety of users. For organizations that have been granted a photocopy license by the $\mathrm{CCC}$, a separate system of payment has been arranged.

Trademark Notice: Product or corporate names may be trademarks or registered trademarks, and are used only for identification and explanation without intent to infringe.

Visit the Taylor \& Francis Web site at

http://www.taylorandfrancis.com

and the CRC Press Web site at

http://www.crepress.com

\section{Library of Congress Cataloging-in-Publication Data}

\section{Thomson, Brian S.}

Symmetric properties of real functions / Brian S. Thomson.

p. cm. -- (Monographs and textbooks in pure and applied mathematics; 183)

Includes bibliographical references and index.

ISBN: 0-8247-9230-0 (acid-free)
1. Symmetric functions.
I. Title.
II. Series.

QA212.T49 1994

515'.83--dc20 


\section{Preface}

The title of this work is taken from the opening paragraph of an article by Mary Weiss [306]:

"It is a familiar fact that symmetric properties of real functions play an important role in a number of problems. This is particularly true of problems in the theory of trigonometric series. The first symmetric derivative (Lebesgue's derivative), the second symmetric derivative (Schwarz's derivative) and their generalizations are familiar notions in the theory of trigonometric series, and the investigation of their properties is a legitimate topic in Real Variables."

By the symmetric properties of real functions we shall mean properties arising from the expression

$$
f(x+t)=\frac{1}{2}[f(x+t)+f(x-t)]+\frac{1}{2}[f(x+t)-f(x-t)]
$$

which defines the even and the odd parts of the function $f$ at the point $x$. For example the continuity and differentiability properties of $f$ can be analyzed in some cases by studying those properties separately in the odd and even parts. Thus we might be led to an investigation of "even continuity" and "odd continuity", and of "even differentiability" and "odd differentiability". This then focuses in on the expressions

$$
f(x+t)-f(x-t) \quad \text { and } \quad f(x+t)+f(x-t)-2 f(x) .
$$

These are two aspects of a single entity; there are many parallels and no study of just one side is quite complete without the other.

It is well known by now that such ideas enjoy an important role in a variety of problems. In the study of trigonometric series in particular various symmetric derivatives come into play in an entirely natural way. The investigation of such notions has become a separate and recognizable topic of study in Real Variables. Larson [173], in an influential survey article, has called it "symmetric real analysis".

The history of this topic stretches back a century and a half and includes contributions from the most important figures in analysis. In every decade of 
this century one can find articles addressing such ideas. Perhaps the most fertile periods have been stimulated by three main influences. Above all Zygmund, who has impacted on so much of the analysis of this century, had long maintained an interest in the differentiability properties of real functions. He and his students have made major advances especially with regard to the symmetric structure of functions. The early years of Fundamenta Mathematicae saw some considerable interest in symmetric problems and dozens of papers and problems addressed such issues; many of the most famous analysts of the period asked or answered symmetric questions in its pages. More recently in my own time the Real Analysis Exchange once more introduced a host of readers to this symmetric literature and its unsolved problems; that the last two decades has produced so much new material in this topic is largely due to the energy and vitality of this journal.

In order to preserve the spirit of the early researches I have tried to present an account that pays attention to the history of the subject. Thus the early chapters present material that is not necessarily stated in the greatest generality and that is proved in mostly the original way. A reading of only the first two chapters affords then a classical view of the subject and its problems together with a perspective on how the ideas and techniques first evolved. Later material will tidy up the loose ends, prove the best versions of theorems and introduce simpler and more powerful techniques.

The background expected of the reader is just the usual assortment of real analysis concepts such as measure, category, density. The ideal reader has already become familiar with the arcana of the ordinary and approximate derivatives through such standard treatises as Saks [251] and Bruckner [18]. The present work is intended to extend many of these ideas to the symmetric setting where mostly different techniques and methods are required. While parts of the early chapters and selections from the later may serve to introduce the curious reader to this subject the main intent has been to provide a reference work for researchers in this area so that they may more easily find out what is known and where it has appeared. For this reason it is hoped that what is presented represents a more or less complete picture of the work done.

Chapter 1 begins the study by centering on the classical symmetric derivatives. The most important result presented here is due to Khintchine and is given as Theorem 1.23 with its original proof. Rather more elementary material appears too in order to fill out the necessary background to our study. In particular we learn most of the basic properties of the first and second symmetric derivatives in this chapter.

Chapter 2 develops the continuity properties of functions satisfying some kind of symmetric growth condition. The centerpiece of this chapter is a single important classical study of symmetric properties of real functions by Charzyński. This represents the first significant study of the continuity properties of functions that satisfy some kind of even or odd symmetry condition. 
The rest of this chapter continues this theme in a number of its forms. Taken together with Chapter 1 this might be considered a brief introduction to the world of symmetric derivatives, symmetric continuity and like concepts. The reader who wishes to go on from here will find a more comprehensive treatment of these kind of ideas.

In Chapter 3 a variety of covering results are obtained that help to unify and clarify the arguments used in this subject.

Chapter 4 begins a systematic study of the regularity properties of functions that satisfy some kind of even symmetric condition. A large literature is surveyed mostly with proofs.

Chapter 5 is devoted to monotonicity and convexity theorems as they arise from the first or second symmetric derivative.

Chapter 6 does for the odd symmetry conditions what Chapter 4 has done for the even conditions. This is a rather complete survey of what is known about the regularity properties of functions that satisfy some kind of odd symmetric growth condition.

Chapter 7 returns to the derivative itself. We have defined a number of symmetric derivatives and this material attempts to survey the nature of the results so far known.

Chapter 8 introduces the variational theory associated with the first and second symmetric derivatives.

Chapter 9 concludes the story with an account of the various symmetric integrals. A primary motivation is the solution of the coefficient problem for trigonometric series.

We include an appendix as a location for material that is needed in the main body of the work but which would be cumbersome to include directly. It is hoped that for the most part the present material is sufficiently inclusive to save the reader numerous trips to the library for source material. Even so there have been plenty of places throughout where proofs have had to be omitted and the original sources should be consulted. Of course, for any serious student of this topic, we should insist that many of the original authors (notably Charzyński, Denjoy, Khintchine, Marcinkiewicz, Mazurkiewicz, Sierpinski and Zygmund) be read in any case!

The problems that are included at the end of the book are, apparently, at the time of writing open. Some of these have an impeccable and legitimate pedigree and will undoubtedly require new methods and insights. Many are just questions that appeared to be unanswered at the time of writing; they may succumb to standard methods.

Our subject has had a long history and many results are now known in their best form. There is much of this subject which can be enshrined now as "knowledge". Thus this monograph is not a report on a rapidly changing field; even so, however, a glance at the bibliography will show that research carries on in this area and it is clear that research will always continue even as the job 
of mining the results becomes tougher and more demanding. As far as I can tell it is a report on the current state of our knowledge in this area and should not obsolete itself except in small portions.

My main thanks are due to the legions of real analysts who have contributed to and deepened our understanding of this subject. More narrowly I would like to thank readers of preliminary versions for their suggestions for improvements, in particular Marshall Ash, Michael Evans, Chris Freiling, Paul Humke and Lee Larson all of whom have been major players in this area and whose comments have been invaluable. My thanks as well go to Hongjian Shi for a careful and detailed reading of several chapters. The manifold faults that remain are exclusively mine.

Brian S. Thomson 


\section{Contents}

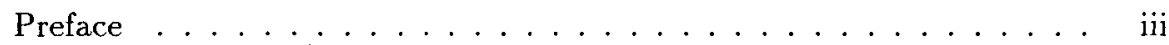

1 THE DERIVATIVE $\ldots \ldots \ldots \ldots \ldots \ldots \ldots$

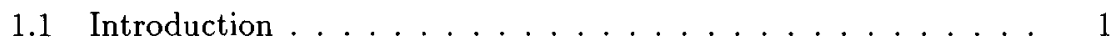

1.2 Even and Odd Properties................. . 2

1.2.1 The Even and Odd Parts of a Function . . . . . . . . . 2

1.2.2 Even and Odd Properties of a Function . . . . . . . . . 3

1.2.3 Higher Order Symmetric Differences . . . . . . . . . 4

1.3 Elementary Considerations . . . . . . . . . . . . . 5

1.4 Riemann's Theorems . . . . . . . . . . . . . . . 8

1.5 Schwarz Theorem . . . . . . . . . . . . . . . . 11

1.6 Auerbach Theorem . . . . . . . . . . . . . . . . . 13

1.7 Borel Symmetric Derivative . . . . . . . . . . . . . . 15

1.8 Approximate Symmetric Derivative . . . . . . . . . . . 17

1.9 Higher Order Symmetric Derivatives . . . . . . . . . . 18

1.10 Khintchine Theorem . . . . . . . . . . . . . . 20

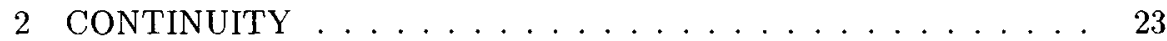

2.1 Introduction . . . . . . . . . . . . . . 23

2.2 Even and Odd Continuity . . . . . . . . . . . 24

2.2.1 Stein-Zygmund Theorem . . . . . . . . . . . 25

2.2.2 Even Version of Stein-Zygmund Theorem . . . . . . 27

2.2 .3 A Reduction Theorem . . . . . . . . . . . . . . . . 28

2.3 Charzyński Theorem . . . . . . . . . . . . . . . 32

2.3.1 Charzyński's Lemma . . . . . . . . . . . . . . . . . 32

2.3.2 $D(f)$ is Nowhere Dense. . . . . . . . . . . . . . 33

2.3.3 $D(f)$ is Denumerable. . . . . . . . . . . 35

$2.3 .4 D(f)$ is Scattered. . . . . . . . . . . . 38

2.3.5 The Even Analogue of Charzyński's Theorem . . . . . 40

2.4 Mazurkiewicz-Auerbach Theorem . . . . . . . . . . . . . 41

2.5 Wolibner Theorem. . . . . . . . . . . . . . . . . . . . 41

2.5.1 A Monotonicity Theorem . . . . . . . . . . . . . . . 44 
2.6 Jurek-Szpilrajn Example . . . . . . . . . . . . . . . . . 45

2.7 Pesin-Preiss Theorem . . . . . . . . . . . . . . . . . 46

2.8 Local Symmetry. . . . . . . . . . . . . . . . . . . . . . . . . . . . . . . . . .

2.8 .1 Points of Local Symmetry . . . . . . . . . . . . . . . . 50

2.9 Points of Discontinuity ....................... 51

2.9.1 Theorem of Tran ............... 56

2.9.2 Theorems of Ponomarev and Chlebík . . . . . . . . 56

2.9.3 Points of Even/Odd Continuity .......... 59

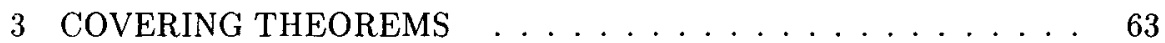

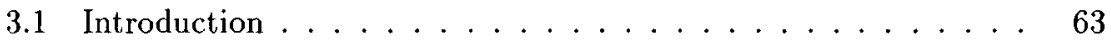

3.2 Symmetric Covering Relations . . . . . . . . . . . . . 66

3.3 Basic Covering Theorems . . . . . . . . . . . . . . . 68

3.3 .1 Uniformization ................. 69

3.3.2 Charzyński's Covering Theorems . . . . . . . . . . 69

3.3.3 Fundamental Covering Theorem for Full Symmetric Covers 71

3.3.4 Other Variants ............... . . 75

3.3.5 Freiling's Negligent Version . . . . . . . . . . . 76

3.4 Khintchine Covering Theorem . . . . . . . . . . . . . 77

3.5 Uher Covering Lemma . . . . . . . . . . . . . . . . . . 79

3.6 Approximate Symmetric Covering Relations . . . . . . . . . . . 83

3.6.1 A Lemma for Measurable Covering Relations. . . . . . . 84

3.6.2 Analysis of the Covering Properties . . . . . . . . . . 85

3.6.3 Partitioning Theorem ....................... 88

3.7 2-Interval Partitions . . . . . . . . . . . . . . . 97

3.7.1 Rectangles and 2-Intervals . . . . . . . . . . . 98

3.7.2 Geometrical Arguments ... . . . . . . . . . . . 101

3.7.3 The Covering Theorem ............... 107

3.7.4 Partitions into Squares .............. 109

4 EVEN PROPERTIES . . . . . . . . . . . . . . 111

4.1 Introduction . . . . . . . . . . . . . . . . 111

4.2 Midpoint-Linear Functions . . . . . . . . . . . . . . . . 112

4.2.1 Additive Functions . . . . . . . . . . . . . . . 113

4.2.2 Hamel Theorem . . . . . . . . . . . . . . . 115

4.2.3 Close to Midpoint-Linear Functions . . . . . . . . . . . . 116

4.2.4 Locally Midpoint-Linear Functions . . . . . . . . . . . 118

4.3 Midpoint-Convex Functions . . . . . . . . . . . . . . . 119

4.3.1 Theorem of Blumberg-Sierpiński . . . . . . . . . 120

4.3.2 Convex Sets, Anticonvex Sets . . . . . . . . . . . 122

4.4 Uniform Smoothness Conditions . . . . . . . . . . . . . 123

4.4.1 Modulus of Continuity ............... 124

4.4.2 Theorem of Anderson and Pitt ........... 127 
4.4.3 Continuously Differentiable Functions . . . . . . . . . . . 127

4.4.4 Theorem of M. Weiss and Zygmund . . . . . . . . . . 129

4.4.5 Almost Nowhere Differentiable Functions . . . . . . . 131

4.4.6 Monotonic, Uniformly Smooth Functions . . . . . . . 133

4.4.7 Some Examples . . . . . . . . . . . . . . . . 134

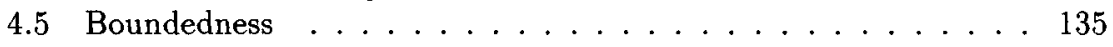

4.6 Symmetric Functions . . . . . . . . . . . . . . . . . . . . 137

4.6.1 Continuity Properties of Symmetric Functions . . . . . . 138

4.6 .2 Baire Class ... . . . . . . . . . . . . 141

4.6.3 Approximately Symmetric Functions . . . . . . . . . 143

4.6.4 Points of Symmetry . . . . . . . . . . . . . 146

4.6.5 Points of Approximate Symmetry . . . . . . . . . . . . 147

4.6.6 $L_{p}$-Symmetric Functions . . . . . . . . . . . . . . . . 148

4.6.7 Determining Sets of Symmetric Functions . . . . . . 148

4.6.8 Derivates of Symmetric Functions . . . . . . . . . . . . 150

4.6.9 Typical Symmetric Functions . . . . . . . . . . . . . 151

4.7 Quasi-Smooth Functions . . . . . . . . . . . . . . 152

4.7.1 Continuity Properties . . . . . . . . . . . . . 152

4.7.2 A Nowhere Differentiable Quasi-Smooth Function . . . . 153

4.7.3 Derivates of Quasi-Smooth Functions . . . . . . . . 153

4.7.4 A Tauberian Theorem ............. 156

4.8 Smooth Functions . . . . . . . . . . . . . . . . . . . . . . . . . . . . . . . . . . . .

4.8.1 Elementary Properties . . . . . . . . . . . . . 159

4.8.2 Continuous, Smooth Functions . . . . . . . . . . . . 161

4.8.3 Makarov's Theorems . . . . . . . . . . . . . . 164

4.8.4 Measurable, Smooth Functions ........... 165

4.8.5 Neugebauer's Example . . . . . . . . . . . . . . 166

4.8.6 Neugebauer Alternative . . . . . . . . . . . . 167

4.8.7 Approximately Smooth Functions . . . . . . . . . 169

$4.8 .8 L_{p}-$ Smooth Functions . . . . . . . . . . . . . . 170

4.8.9 Discontinuities of $L_{p}$-Smooth Functions . . . . . . . . . . 171

4.8.10 Discontinuities of Approximately Smooth Functions . . . 172

4.9 Super-Smooth Functions . . . . . . . . . . . . . . . . . 174

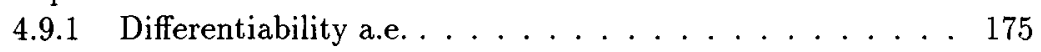

4.9.2 Integral of Marcinkiewicz . . . . . . . . . . . . . 177

4.9.3 Theorem of Denjoy . . . . . . . . . . . . 178

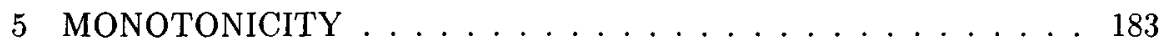

5.1 Introduction . . . . . . . . . . . . . . . 183

5.2 Some Basic Monotonicity Theorems . . . . . . . . . . . . . . 184

5.3 Splattered and Scattered Versions . . . . . . . . . . . 185

5.3.1 Freiling Semi-Scattered Theorem .......... . 188

5.3.2 Freiling Scattered Theorem ... . . . . . . . . . 190 
5.3.3 Freiling Proof of Charzyński Theorem . . . . . . . . . . 191

5.3.4 The Even Analogue of Charzyniski Theorem . . . . . . 194

5.4 Evans-Larson Theorem . . . . . . . . . . . . . . . . . . . 196

5.5 Mean Value Theorems . . . . . . . . . . . . . . . . . 198

5.6 Freiling-Rinne Theorem . . . . . . . . . . . . 200

5.7 Convexity Theorems . . . . . . . . . . . . . . 202

5.7.1 Further Convexity Theorems . . . . . . . . . . . 205

5.8 Monotonicity Appendix . . . . . . . . . . . . . . . . . 210

5.8.1 A Brief History of Symmetric Monotonicity Theorems . . 210

6 ODD PROPERTIES . . . . . . . . . . . . . . . . . 213

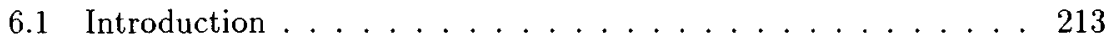

6.2 Symmetry . . . . . . . . . . . . . . . . . . 214

6.2 .1 Exact Symmetry . . . . . . . . . . . . . . . 214

6.2 .2 Essential Symmetry . . . . . . . . . . . . . . 218

6.2.3 Exact Local Symmetry Everywhere . . . . . . . . . . 222

6.2 .4 Points of Local Symmetry of a Set . . . . . . . . . 225

6.2.5 Points of Local Symmetry of a Function . . . . . . . . 229

6.3 Symmetric Monotonicity . . . . . . . . . . . . . . . . . 231

6.3.1 Everywhere Symmetric Increase . . . . . . . . . . 232

6.3.2 Points of Symmetric Increase . . . . . . . . . . . 232

6.3.3 Porosity Properties . . . . . . . . . . . . . . . 234

6.4 Symmetric Continuity . . . . . . . . . . . . . . . 237

6.4 .1 Theorem of Uher . . . . . . . . . . . . . . . . . 238

6.4.2 Symmetrically Continuous Functions . . . . . . . . 241

6.4.3 Discontinuities of Symmetrically Continuous Functions . 242

6.4.4 Weak Symmetric Continuity . . . . . . . . . . . 242

6.5 Chlebík Theorem . . . . . . . . . . . . . . . . 24 245

6.6 Boundedness . . . . . . . . . . . . . . . . 245

6.7 Symmetric Lipschitz Conditions . . . . . . . . . . . . . 247

7 THE SYMMETRIC DERIVATIVE $\ldots \ldots \ldots \ldots \ldots$

7.1 Introduction . . . . . . . . . . . . . . . . . . . . 249

7.2 Extreme Symmetric Derivatives . . . . . . . . . . . 250

7.2.1 Relations Among the Derivates . . . . . . . . . . . 251

7.2 .2 Measurability of Derivates . . . . . . . . . . . . 255

7.2 .3 Nonmeasurable Derivates . . . . . . . . . . . . . . . . 255

7.2.4 Baire Class of Symmetric Derivates . . . . . . . . . 256

7.2.5 Borel Measurability of Derivates . . . . . . . . . . . 257

7.2.6 Porosity Relations for Symmetric Derivates . . . . . . . 257

7.2.7 Denjoy Relations for Symmetric Derivates . . . . . . 258

7.3 Symmetric Derivatives . . . . . . . . . . . . . . . 262

7.3.1 Baire Class of Symmetric Derivatives . . . . . . . . . 262 
7.3.2 Symmetric Differentiability . . . . . . . . . . . . 264

7.3.3 Theorem of Belna, Evans and Humke . . . . . . . . . . 265

7.3.4 Example of Foran-Ponomarev . . . . . . . . . . . 266

7.3.5 Points of Non Symmetric-Differentiability . . . . . . 266

7.3.6 Infinite Symmetric Derivatives . . . . . . . . . . . . 268

7.3.7 Steep Infinite Derivatives . . . . . . . . . . . . . . . 269

7.3 .8 Zero Symmetric Derivative . . . . . . . . . . . . . . 272

7.3.9 Stationary Sets for Symmetric Derivatives . . . . . . . 273

7.3.10 Larson's Primitive . . . . . . . . . . . . . . . 273

7.3.11 The Range of Symmetric Derivatives . . . . . . . . 276

7.4 Approximate Symmetric Derivative . . . . . . . . . . 278

7.4.1 Baire Class of Approximate Symmetric Derivative . . . . 278

7.4.2 Baire Class of Approximate Symmetric Derivates . . . . 280

7.4.3 Measurability of the Approximate Symmetric Derivative 283

7.4.4 Approximate Version of Khintchine Theorem . . . . . . . 285

7.4.5 Relations for Monotone Functions . . . . . . . . . . . 286

7.4.6 Typical Continuous Functions . . . . . . . . . . . 287

7.5 Borel Symmetric Derivative . . . . . . . . . . . 288

8 SYMMETRIC VARIATION . . . . . . . . . . . . . . 293

8.1 Introduction . . . . . . . . . . . . . . . . . . 293

8.2 First Order Symmetric Variation . . . . . . . . . . . 294

8.2 .1 Zero Variation . . . . . . . . . . . . . . . . . . 295

8.2 .2 Finite Variation . . . . . . . . . . . . . . . . . 297

8.2 .3 Variation and Symmetric Derivates . . . . . . . . 300

8.3 Second Order Variation. . . . . . . . . . . . . 302

8.3.1 Functions on Rectangles and 2-Intervals . . . . . . . . 302

8.3 .2 Symmetric Increments . . . . . . . . . . . . . . . . 304

8.3.3 Variational Definitions . . . . . . . . . . . . . 306

8.3.4 Basic Properties . . . . . . . . . . . . . . . . . . . . 309

8.3.5 Variation of a Continuous Function . . . . . . . . 313

8.3 .6 Zero Variation . . . . . . . . . . . . . . . . . . . . . . 314

8.3.7 Differentiation and Variation. . . . . . . . . . . 316

8.3 .8 Finite Variation . . . . . . . . . . . . . . . . . 320

8.3.9 Generalization of Schwarz Theorem. . . . . . . . . . 321

8.4 A Theorem of Denjoy . . . . . . . . . . . . . . . 322

8.5 An Example of Skvorcov . . . . . . . . . . . . . . . 322

9 SYMMETRIC INTEGRALS . . . . . . . . . . . . . . . 323

9.1 Introduction . . . . . . . . . . . . . . . . 323

9.1.1 Integrals from Derivatives. . . . . . . . . . . . 325

9.2 The Ordinary Symmetric Integral _ . . . . . . . . . . . . . 329

9.2 .1 Preliminaries . . . . . . . . . . . . . . . . . . . . . 329 
9.2.2 A Symmetric Newton Integral . . . . . . . . . . . 330

9.2.3 An Elementary Application to Trigonometric Series . . . 335

9.2.4 A Symmetric Totalization . . . . . . . . . . . . . 336

9.2.5 A Symmetric Variational Integral . . . . . . . . . . . 341

9.2.6 Symmetric Absolute Continuity . . . . . . . . . . . 346

9.2.7 A Further Application to Trigonometric Series . . . . . . 351

9.2.8 A Symmetric Perron Integral ... . . . . . . . . . 352

9.2.9 A Symmetric Riemann Integral . . . . . . . . . . . 354

9.2.10 Variational Characterization of the $\left(\mathrm{R}_{s}^{1}\right)$-Integral . . . 357

9.2.11 Lusin Type Characterization . . . . . . . . . . . . . 361

9.3 The Approximate Symmetric Integral . . . . . . . . . . . . . . . 362

9.3.1 Preliminaries ................ . . 362

9.3.2 An Approximate Symmetric Newton Integral . . . . . . . 363

9.3.3 An Application to Trigonometric Series . . . . . . . . . . 366

9.3.4 An Approximate Symmetric Variational Integral . . . . . 368

9.3.5 Approximate Symmetric Absolute Continuity . . . . . . 374

9.3.6 An Approximate Symmetric Perron Integral . . . . . . . 378

9.3.7 An Approximate Symmetric Riemann Integral . . . . . . 380

9.3.8 Applications to Trigonometric Series . . . . . . . . . . . 383

9.4 Second Order Symmetric Integrals . . . . . . . . . . . . . . 388

9.4.1 A Second Order Symmetric Newton Integral . . . . . . . 389

9.4.2 The Definitions and an Integrability Criterion ..... . 391

9.4.3 Properties of the Integral . . . . . . . . . . . . . . . 394

9.4 .4 Integration and Variation . . . . . . . . . . . 397

9.4.5 An Application to Trigonometric Series . . . . . . . . . . 398

9.5 Incompatibilities . . . . . . . . . . . . . . 399

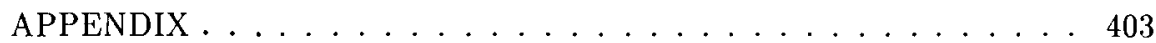

A.1 Scattered Sets . . . . . . . . . . . . . . . 403

A.2 Scattered Baire theorem . . . . . . . . . . . . . 406

A.3 A Density Computation . . . . . . . . . . . . . . . . 408

A.4 Density Points. . . . . . . . . . . . . . . . . . . 409

A.5 Category Density Points . . . . . . . . . . . . . . . 410

A.6 Hamel Bases . . . . . . . . . . . . . . . . . . . . . . 411

A.7 Weak Quasi-Continuity . . . . . . . . . . . . . 412

A.8 Weak Approximate Continuity . . . . . . . . . . . . . . 413

A.9 Baire Class . . . . . . . . . . . . . . . . . 414

A.10 Goffman Theorem . . . . . . . . . . . . . . . . 414

A.11 Measurability . . . . . . . . . . . . . . . . . . 415

A.12 The Baire Property . . . . . . . . . . . . . . . 417

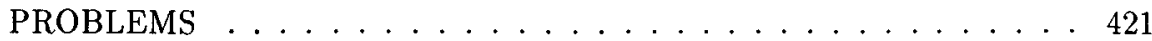


References ........................ 427

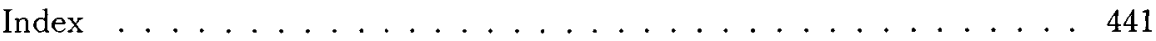



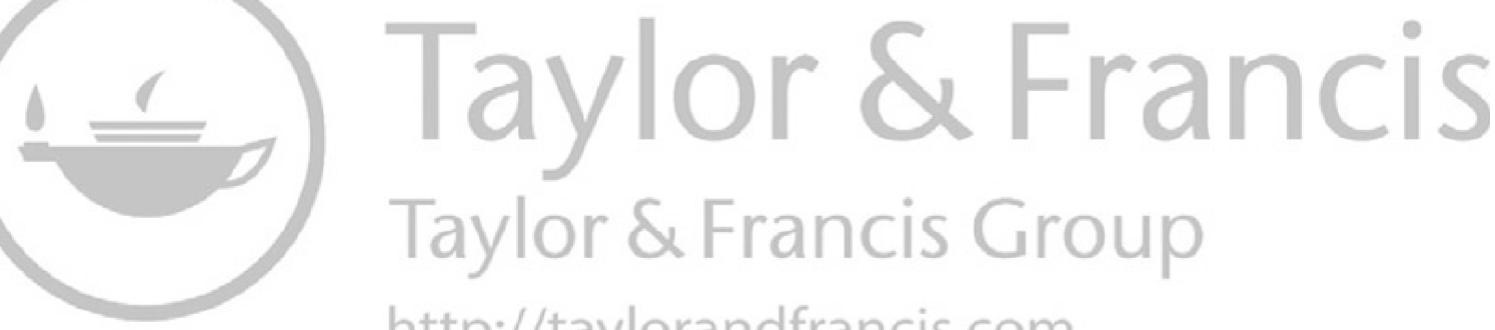

http://taylorandfrancis.com 


\section{SYMMETRIC \\ PROPERTIES \\ OF REAL \\ FUNCTIONS}



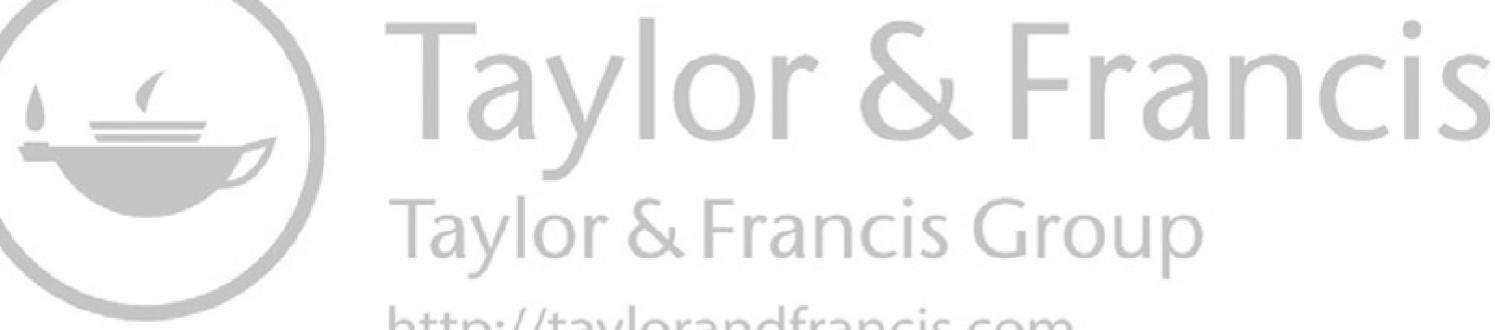

http://taylorandfrancis.com 


\section{1}

\section{The Derivative}

\subsection{Introduction}

The first and second symmetric derivatives of a function $f$ are defined by the expressions

$$
\begin{gathered}
\mathrm{SD} f(x)=\lim _{t \rightarrow 0} \frac{f(x+t)-f(x-t)}{2 t} . \\
\mathrm{SD}_{2} f(x)=\lim _{t \rightarrow 0} \frac{f(x+t)+f(x-t)-2 f(x)}{t^{2}} .
\end{gathered}
$$

The first of these is, on occasion, called Lebesgue's derivative and the second Riemann's derivative or Schwarz's derivative. We begin our work with some classical studies of these derivatives. Although our concerns are broader than this, these studies provide us the opportunity to present many of the main themes and will lead us into the subject.

The first question that would obviously arise in a study of the symmetric derivatives is to ask how far properties of ordinary derivatives extend to symmetric derivatives. This is a natural starting point for our investigation and is the starting point taken by Khintchine [151] in 1927. This leads, by relatively familiar methods, to a number of elementary observations. We supplement this with several other important classical, but essentially elementary, results of Schwarz, Mazurkiewicz [197] and [198], and Auerbach [9]. The applications to trigonometric series that appear in Sections 1.4 and 1.7 remain among the central reasons why the subject has attracted attention.

The main result in Khintchine's fundamental paper, the first really technically interesting result in the subject, appears in Section 1.10. This asks the question: does the existence of the symmetric derivative of a function $f$ on a set $E$ say anything about the ordinary differentiability properties of $f$ on that 
set? The answer to this question will introduce us to some of the more subtle geometric arguments needed to study effectively this derivative in particular and symmetric properties of real functions in general.

\subsection{Even and Odd Properties}

\subsubsection{The Even and Odd Parts of a Function}

A function $f$ is even if $f(t)=f(-t)$ everywhere; a function $f$ is odd if $f(t)=-f(-t)$ everywhere. These conditions represent symmetries in the graph of the function, in the first case symmetry about the $y$-axis and in the second symmetry about the origin.

At any point $x$ and for any function $f$ we can study these symmetries in the ordinary difference

$$
\Delta f(x, t)=f(x+t)-f(x) .
$$

This difference can be written as

$$
f(x+t)-f(x)=\frac{f(x+t)-f(x-t)}{2}+\frac{f(x+t)+f(x-t)-2 f(x)}{2} .
$$

We shall employ the notations

$$
\Delta^{1}{ }_{s} f(x, t)=f(x+t)-f(x-t)
$$

and

$$
\Delta^{2} f(x, t)=f(x+t)+f(x-t)-2 f(x)
$$

occasionally to represent these odd and even increments. They are known as the first symmetric difference and second symmetric difference for the function $f$.

These expressions appear in a variety of studies. For example the connection of these ideas with trigonometric series is by now well known. For instance if

$$
a_{0} / 2+\sum_{n=1}^{\infty}\left(a_{n} \cos n x+b_{n} \sin n x\right)
$$

is the Fourier series of a function $f$ then the convergence behavior or the summability behavior of the series at a point $x$ or of the differentiated series (differentiated an even number of times) depends very much on properties of the function

$$
t \rightarrow f(x+t)+f(x-t)-2 f(x)
$$

near the point $t=0$, that is to say on properties of the even difference. For a well known example, a theorem of Dini (see [313, Theorem 6.1, p. 52]) asserts 
that the Fourier series of a function $f$ converges at a point $x$ to the sum $f(x)$ provided that the integral

$$
\int_{0}^{\pi} \frac{|f(x+t)+f(x-t)-2 f(x)|}{t} d t
$$

is finite.

The odd difference of the function plays a dual role. For example the behavior of the conjugate Fourier series for $f$ or of the differentiated series (differentiated an odd number of times) depends on the odd part of $f$ at $x$, that is depends on properties of the function

$$
t \rightarrow f(x+t)-f(x-t)
$$

near the point $t=0$. Again, for example, the result analogous to that of Dini cited above is that of Pringsheim (again see [313, Theorem 6.1, p. 52]) asserting that the conjugate Fourier series of a function $f$ converges at a point $x$ provided that the integral

$$
\int_{0}^{\pi} \frac{|f(x+t)-f(x-t)|}{t} d t
$$

is finite.

An accumulation of results of this kind has led to a study of such properties for their intrinsic interest. This offers some preliminary motivation for a study of even and odd properties of functions. In this chapter we begin by an investigation of the symmetric derivatives and follow where they lead. The even and odd structure of these two derivatives is, of course, obvious.

\subsubsection{Even and Odd Properties of a Function}

The ordinary difference can be written usefully as

$$
\Delta f(x, t)=\frac{1}{2} \Delta^{1}{ }_{s} f(x, t)+\frac{1}{2} \Delta^{2}{ }_{s} f(x, t)
$$

using these odd and even differences. It is natural in many contexts to examine the continuity and differentiability properties of $f$ by studying those properties in these two parts. This follows a well known and useful tradition in analysis resulting in notions such as right and left hand continuity, upper and lower semicontinuity, right/left and upper/lower derivates (Dini derivatives) and so on. The goal is to split a property into smaller conditions allowing separate analysis. Thus we might be led to an investigation of "even continuity" and "odd continuity", and of "even differentiability" and "odd differentiability".

The continuity of a function $f$ at a point $x$, that is the requirement that $\Delta f(x, t) \rightarrow 0$ as $t \rightarrow 0$, is equivalent to requiring both $\Delta^{1}{ }_{s} f(x, t) \rightarrow 0$ and $\Delta^{2}{ }_{s} f(x, t) \rightarrow 0$. This might split into two "semi-continuity" conditions. It seems natural to say that a function $f$ is even continuous at $x$ if $\Delta^{2}{ }_{s} f(x, t) \rightarrow 0$ and 
is odd continuous at $x$ if $\Delta^{1}{ }_{s} f(x, t) \rightarrow 0$. The terminology, however, has not evolved in any such systematic manner. Continuity of the odd part of $\Delta f(x, t)$ is known as symmetric continuity at $x$ while $f$ is said to be symmetric at $x$ if the even part is continuous. In Chapter 2 we study extensively such notions.

Similarly, differentiability of a function $f$ at a point $x$ is equivalent to the existence of both of the limits

$$
\lim _{t \rightarrow 0} \frac{\Delta^{1} f(x, t)}{t} \text { and } \lim _{t \rightarrow 0} \frac{\Delta^{2}{ }_{s} f(x, t)}{t} .
$$

The derivative of the odd part is exactly the symmetric derivative SD $f(x)$ of the function $f$ at the point $x$. On the other hand differentiability of the even part is easily checked to be equivalent to the requirement that $\Delta^{2}{ }_{s} f(x, t)=o(t)$, i.e. that

$$
\lim _{t \rightarrow 0} \frac{f(x+t)+f(x-t)-2 f(x)}{t}=0,
$$

and this condition is usually called the smoothness of the function $f$ at the point $x$. (This notion was first considered by Riemann in his famous memoir on trigonometric series; the terminology is due to Zygmund.) The second symmetric derivative $\mathrm{SD}_{2} f(x)$ now can be viewed as a kind of second order even derivative.

In order to catalogue our many results in this work we shall use the following language. By an odd property of a function $f$ we shall mean some property expressed in terms of the odd symmetric difference $\Delta^{1}{ }_{s} f(x, t)$ and by an even property of a function $f$ we shall mean some property expressed in terms of the even symmetric difference $\Delta^{2}{ }_{s} f(x, t)$.

Note that a function is even if and only if its odd part vanishes. Thus the even symmetric structure of a function is expressed as an odd property. Similarly the odd symmetry of a function is expressed as an even property. Thus in general we can think of the study of the even/odd properties of a function as promoting the odd/even symmetric structure of the function.

\subsubsection{Higher Order Symmetric Differences}

The two symmetric differences $\Delta^{1}{ }_{s} f(x, t)$ and $\Delta^{2}{ }_{s} f(x, t)$ can be considered as the odd and even parts of $\Delta f(x, t)$. More commonly they are referred to as the first and second symmetric differences of $f$ at $x$ and our notation reflects this.

The terminology indicates the fact that the second difference can be viewed as an iteration of the first. Write

$$
g(x)=\Delta^{1}{ }_{s} f(x, h)=f(x+h)-f(x-h) .
$$

A direct and elementary computation shows that

$$
\begin{aligned}
& \Delta^{1}{ }_{s} g(x, h)=g(x+h)-g(x-h) \\
& \quad=\quad=f(x+2 h)+f(x-2 h)-2 f(x)=\Delta^{2}{ }_{s} f(x, 2 h) .
\end{aligned}
$$


One can evidently continue in this fashion forming inductively a hierarchy of symmetric differences. A notation (cf. [313, Vol. II, p. 61]) that places all of these in the same context is to write instead

$$
\Delta_{s}^{n} f(x, t)=\sum_{i=0}^{n}(-1)^{i} \frac{n !}{(n-i) ! i !} f\left(x+i t-\frac{1}{2} n t\right) .
$$

We mostly avoid using any such notation and we write out explicitly the even and odd expressions as needed.

In our study we confine our attention to what might be called the symmetric structure of a function, namely properties expressible in terms of the even and odd differences. There is, by now, a rich literature devoted to higher order symmetric derivatives and higher order smoothness conditions. Since these properties do not directly express properties of the odd and even parts of a function we can restrict the scope of our work by not reporting on this activity.

\subsection{Elementary Considerations}

The symmetric derivative has been defined as a kind of odd derivative. In any discussion of generalized derivatives, of which the symmetric derivative may be considered one of the simplest, it is natural to ask to what extent the usual rules of derivation apply. The first formal presentation of the symmetric derivative along these lines was given by Khintchine [151] in an early paper in Fundamenta Mathematicae. The elementary observations in this section are from that paper.

A simple example suffices to show that the properties of the symmetric derivative are very different from those of the ordinary derivative. For example the function $f(x)=|x|$ is everywhere symmetrically differentiable with

$$
\operatorname{SD} f(x)= \begin{cases}1 & \text { if } x>0 \\ -1 & \text { if } x<0 \\ 0 & \text { if } x=0\end{cases}
$$

and this dispenses with a number of the simpler properties that might be expected. The symmetric derivative can evidently exist when the ordinary derivative does not and the derivative itself need not have the intermediate value property (Darboux property). The characteristic function of any finite set has an everywhere vanishing symmetric derivative, but is not itself constant.

The elementary formula

$$
\frac{f(x+h)-f(x-h)}{2 h}=\frac{1}{2}\left\{\frac{f(x+h)-f(x)}{h}+\frac{f(x-h)-f(x)}{-h}\right\}
$$

illustrates the symmetric derivative as the average of the two one-sided derivatives at points where both exist. Khintchine [151] sketches an example to show 
that Rolle's theorem cannot hold: construct a continuous function $f$ that vanishes at 0 and 1 , has a positive derivative on $\left(0, \frac{1}{2}\right)$, a negative derivative on $\left(\frac{1}{2}, 1\right)$, and such that $f^{\prime}\left(\frac{1}{2}+0\right)=0$ and $f^{\prime}\left(\frac{1}{2}-0\right)=1$. Here although $f(0)=f(1)$ and the symmetric derivative exists everywhere on the interval $(0,1)$ it never vanishes. This example too shows that symmetric derivatives do not necessarily have the Darboux property.

We can summarize this discussion by the following elementary lemmas.

Lemma 1.1 If $f^{\prime}(x)$ exists then so too does $S D f(x)$ and they are equal.

Lemma 1.2 If the one-sided derivatives $f_{+}^{\prime}(x)$ and $f_{-}^{\prime}(x)$ exist then so too does $S D f(x)$ and

$$
\operatorname{SDf}(x)=\frac{f_{+}^{\prime}(x)+f_{-}^{\prime}(x)}{2} .
$$

Lemma 1.3 There is a continuous function $f$ that has a finite symmetric derivative everywhere but such that $S D f(x)$ does not have the Darboux property.

A more interesting problem, which has subtle and curious answers, is to ask how far monotonicity theorems can be carried to this kind of derivative. If a function $f$ has everywhere a positive symmetric derivative then is the function increasing or nearly so? We shall have occasion to return to this problem again but here we shall reproduce an elementary monotonicity theorem from [151, p. 216] with its original proof; by 1927 when Khintchine's paper appeared this theorem would probably have been known. The proof, it should be noted, is merely a minor modification of a well known proof of a monotonicity theorem for the Dini derivatives ( $c f$. [251, pp. 203-204]) and the ideas go back at least to 1878. There is no special use of symmetric arguments here and the proof cannot be considered very informative for us. A full study of monotonicity theorems for the symmetric derivatives appears in Chapter 5.

Theorem 1.4 A continuous function is necessarily increasing in any interval in which its symmetric derivative exists and is positive.

Proof. Let us show that for every pair of points $x_{1}$ and $x_{2}$ in this interval $f\left(x_{1}\right)<f\left(x_{2}\right)$ if $x_{1}<x_{2}$. If not then there are such points with $f\left(x_{1}\right)>f\left(x_{2}\right)$ or $f\left(x_{1}\right)=f\left(x_{2}\right)$. But the latter possibility reduces also to the former because a further point $x_{3}$ in between could be chosen with $f\left(x_{3}\right) \neq f\left(x_{1}\right)$. Let us suppose then that for some pair of points $x_{1}$ and $x_{2}$ with $x_{1}<x_{2}, f\left(x_{1}\right)>f\left(x_{2}\right)$.

Choose any

$$
f\left(x_{1}\right)>c>f\left(x_{2}\right)
$$

and take the point

$$
z=\inf \left\{x \in\left(x_{1}, x_{2}\right): f(x)<c\right\}
$$


which evidently lies on the level $f^{-1}(c)$. For some sequence of points $x_{n} \searrow z$, $f\left(x_{n}\right)<f(z)=c$. The symmetric points $2 z-x_{n}$ on the other side of $z$ must have $f\left(2 z-x_{n}\right) \geq f(z)=c$ and so

$$
f\left(x_{n}\right)-f\left(2 z-x_{n}\right) \leq 0 .
$$

Hence $\operatorname{SD} f(z)$ cannot be positive, and with this contradiction the theorem is established.

As a corollary we deduce that continuous functions with zero symmetric derivatives are constant. Hence functions with identical symmetric derivatives differ by a constant. This was probably first proved in Mazurkiewicz [197].

Corollary 1.5 Two continuous functions having identical (finite) symmetric derivatives everywhere in an interval, must differ by a constant in that interval.

Proof. If $f$ and $g$ have identical (finite) symmetric derivatives everywhere in an interval $(a, b)$ then $\operatorname{SD}(f(x)-g(x))=0$ there. For any $\epsilon>0$ this means that $f(x)-g(x)+\epsilon x$ has a positive symmetric derivative and so, by the theorem, must be increasing; similarly $g(x)-f(x)+\epsilon x$ has a positive symmetric derivative and must also be increasing. As $\epsilon>0$ is arbitrary $f-g$ must be constant as required.

This theorem can be looked at in another useful manner. As we have already observed, there is no Rolle's theorem available for the symmetric derivative and hence no mean value theorem. In its place we have the following assertion which we can view as a quasi-mean value theorem. For more theorems of this type see Chapter 5 .

Corollary 1.6 Let $f$ be continuous in an interval $[a, b]$ and suppose that its symmetric derivative exists at each point in $(a, b)$. Then there are points $\xi_{1}$ and $\xi_{2}$ in $(a, b)$ such that

$$
S D f\left(\xi_{1}\right) \leq \frac{f(b)-f(a)}{b-a} \leq S D f\left(\xi_{2}\right)
$$

Proof. Let $k=(f(b)-f(a)) /(b-a)$ and suppose, contrary to the statement of the corollary that there is no point $a<\xi<b$ for which $\operatorname{SD} f(\xi) \leq k$. Then the function $g(x)=f(x)-k(x-a)$ has a symmetric derivative that is positive in $(a, b)$. By the theorem $g$ is increasing and so $g(b)>g(a)$. This gives $f(b)>f(a)+k(b-a)$ which is a contradiction. It follows that $\operatorname{SD} f(\xi) \leq k$ for at least one $\xi \in(a, b)$. In a similar manner it follows too that $\operatorname{SD} f(\xi) \geq k$ for at least one $\xi \in(a, b)$. 


\subsection{Riemann's Theorems}

Let us pass now to even derivatives. These notions first arose in Riemann's study of trigonometric series. In his investigation of series of the form

$$
f(x)=a_{0} / 2+\sum_{n=1}^{\infty}\left(a_{n} \cos n x+b_{n} \sin n x\right),
$$

where the coefficients converge to zero, he was led to a study of the function

$$
R(x)=a_{0} x^{2} / 4-\sum_{n=1}^{\infty}\left(a_{n} \cos n x+b_{n} \sin n x\right) / n^{2}
$$

obtained by formally integrating the series (1.1) twice. This function $R$ is now called the Riemann function for the series. The properties of the limits

$$
\lim _{h \rightarrow 0+} \frac{R(x+h)+R(x-h)-2 R(x)}{h}
$$

and

$$
\lim _{h \rightarrow 0+} \frac{R(x+h)+R(x-h)-2 R(x)}{h^{2}}
$$

constitute what is commonly called the Riemann theory of trigonometric series (cf. [313, Vol. I, Chapter 9]).

The mere existence of the limit in (1.2) forces that limit to be zero. A function with this property at a point $x$ is said to be smooth at $x$. Riemann's second theorem (Theorem 1.10 below) says that the Riemann function is uniformly smooth.

The limit in (1.3) we have already introduced as the second order symmetric derivative and we use the terminology $\mathrm{SD}_{2} R(x)$ for this derivative. Any study of the first order symmetric derivative must lead soon to a confrontation with the second order symmetric derivative. The genesis of these derivatives in the expression for the even and odd parts of a function alone should suggest this.

This derivative also arises by integrating a first symmetric derivative. If $\operatorname{SD} f\left(x_{0}\right)=c$ then

$$
\frac{f\left(x_{0}+t\right)-f\left(x_{0}-t\right)}{2 t} \rightarrow c
$$

as $t \rightarrow 0$. If $f$ is integrable and $F$ is an indefinite integral of $f$ then $\mathrm{SD}_{2} F\left(x_{0}\right)=c$ as some elementary computations show:

$$
\begin{aligned}
& F\left(x_{0}+h\right)+F\left(x_{0}-h\right)-2 F(x) \\
& \quad=\left(F\left(x_{0}+h\right)-F\left(x_{0}\right)\right)-\left(F\left(x_{0}\right)-F\left(x_{0}-h\right)\right) \\
& =\int_{0}^{h}\left(f\left(x_{0}+t\right)-f\left(x_{0}-t\right)\right) d t \\
& =c h^{2}+\int_{0}^{h}\left(\left[f\left(x_{0}+t\right)-f\left(x_{0}-t\right)\right]-2 c t\right) d t .
\end{aligned}
$$


From this and (1.4) we can deduce

$$
\frac{F\left(x_{0}+h\right)+F\left(x_{0}-h\right)-2 F(x)}{h^{2}} \rightarrow c .
$$

Thus statements about the symmetric derivatives of integrable functions can be lifted up to the primitive function by using the second order symmetric derivative. One advantage here is that the primitive is generally a nicer function than the original function; it is absolutely continuous for example. Of course the method is limited to integrable functions but in earlier times this was not seen as much of a limitation.

As a first step in the study of the second symmetric derivative let us note that if $f^{\prime \prime}(x)$ exists then so too does the second symmetric derivative and both have the same value. Thus this latter derivative is a generalized version of the ordinary second order derivative. By an application of the mean-value theorem of the calculus, the fraction

$$
\frac{f(x+h)+f(x-h)-2 f(x)}{h^{2}}
$$

must have the value

$$
\frac{f^{\prime}(x+k)-f^{\prime}(x-k)}{2 k}
$$

for some $0<k<h$. Letting $h \rightarrow 0, k \rightarrow 0$ we obtain

$$
\frac{f^{\prime}(x+k)-f^{\prime}(x-k)}{2 k} \rightarrow f^{\prime \prime}(x)
$$

which justifies our assertion.

We can summarize these observations as lemmas.

Lemma 1.7 Let $f$ be an integrable function with a symmetric derivative $S D f\left(x_{0}\right)$ at a point $x_{0}$. If $F$ is an indefinite integral of $f$ then $S D_{2} F\left(x_{0}\right)=$ $\operatorname{SD} f\left(x_{0}\right)$.

Lemma 1.8 Let $F^{\prime \prime}\left(x_{0}\right)$ exist. Then $S D_{2} F\left(x_{0}\right)=F^{\prime \prime}\left(x_{0}\right)$.

Riemann's first theorem (Theorem 1.9 below) asserts that the second symmetric derivative of the Riemann function for a trigonometric series recovers the sum of the series at any point at which the series converges.

Theorem 1.9 (Riemann's first theorem) Let $R$ denote the Riemann function for the trigonometric series

$$
a_{0} / 2+\sum_{n=1}^{\infty}\left(a_{n} \cos n x+b_{n} \sin n x\right)
$$


where $a_{n}, b_{n} \rightarrow 0$. If (1.5) converges to a finite sum $s$ at a point $x_{0}$ then $S D_{2} R\left(x_{0}\right)=s$.

Proof. Elementary trigonometric identities and the expression for $R$ lead immediately to the fact that

$$
\begin{aligned}
& \frac{R\left(x_{0}+2 h\right)+R\left(x_{0}-2 h\right)-2 R\left(x_{0}\right)}{4 h^{2}} \\
& =a_{0} / 2+\sum_{n=1}^{\infty}\left(a_{n} \cos n x_{0}+b_{n} \sin n x_{0}\right)\left(\frac{\sin n h}{n h}\right)^{2} .
\end{aligned}
$$

The rest of the proof then is just a matter of computing this limit as $h \rightarrow 0$. Details of this computation appear in [11, Vol. I, Theorem 2, pp. 190-191]. This may also be viewed as a regularity claim for a summability method as in [313, Vol. 1, Theorem 2.4, pp. 319-320].

Theorem 1.10 (Riemann's second theorem) Let $R$ be the Riemann function for a trigonometric series

$$
a_{0} / 2+\sum_{n=1}^{\infty}\left(a_{n} \cos n x+b_{n} \sin n x\right)
$$

with $a_{n}, b_{n} \rightarrow 0$. Then

$$
\lim _{h \rightarrow 0+} \frac{R(x+h)+R(x-h)-2 R(x)}{h}=0
$$

uniformly in $x$.

Proof. This statement too may be viewed as a regularity claim for a summability method as in [313, Vol. I, Theorem 2.8, p. 320]. A more general assertion is proved too in [313, Vol. I, Theorem 2.11, pp. 320-321].

These two theorems illustrate the role of "even" derivatives in the study of trigonometric series. The odd derivatives play a role too. As a sample let us state a theorem due essentially to Fatou and closely related to the two theorems of Riemann.

Theorem 1.11 (Fatou) Suppose that $a_{n}$ and $b_{n}$ are $o(1 / n)$ or, more generally, that

$$
\sum_{k=1}^{n} k \sqrt{a_{k}^{2}+b_{k}^{2}}=o(n) .
$$

Then, if $s_{n}(x)$ are the partial sums of a trigonometric series,

$$
a_{0} / 2+\sum_{n=1}^{\infty}\left(a_{n} \cos n x+b_{n} \sin n x\right)
$$


the series

$$
L(x)=\frac{1}{2} a_{0} x+\sum_{n=1}^{\infty}\left(a_{n} \sin n x-b_{n} \cos n x\right) / n
$$

obtained by a single integration of the series converges uniformly and absolutely to a continuous, uniformly smooth function $L$. Moreover

$$
\frac{L(x+h)-L(x-h)}{2 h}-s_{N(h)}(x) \rightarrow 0
$$

uniformly in $x$ as $h \rightarrow 0$ where $N(h)=[1 / h]$ (the integral part of $1 / h$ ).

Proof. See [313, Vol. I, Theorem 2.16, p. 322].

We should point out that although there is a symmetric derivative (odd derivative) implicit in this assertion the function $L$ is smooth (it has an even derivative) so that should SD $L(x)$ exist it must be simply $L^{\prime}(x)$. Even so the expression in (1.7) makes it clear that it is symmetric differentiation rather than ordinary differentiation that dominates this kind of study.

\subsection{Schwarz Theorem}

One of the most interesting and immediate applications of Riemann's theory of trigonometric series is to the problem of uniqueness. The problem was posed by Heine: can a function have two different representations as the sum of convergent trigonometric series? By subtracting two such series we see that the question reduces to asking whether there can exist a trigonometric series

$$
a_{0} / 2+\sum_{n=1}^{\infty}\left(a_{n} \cos n x+b_{n} \sin n x\right),
$$

converging everywhere to 0 but whose coefficients are not all zero. Heine had shown that for uniform convergence there was uniqueness but recognized that for pointwise convergence the problem was more delicate.

The problem was solved by Cantor [41, pp. 82-83] in a report from 1870. The solution stands as a landmark in the history of mathematics, not so much for the depth of the methods themselves but for the fact that it marked the beginning of a research program for Cantor that culminated in the development of modern set theory and the theory of transfinite ordinals.

Cantor's solution has three simple ingredients. He showed first that if the trigonometric series (1.8) converges everywhere to 0 then the coefficients $a_{n}$, $b_{n} \rightarrow 0$. Take then the Riemann function $R$ for the series; by Riemann's first theorem (Theorem 1.9) $\mathrm{SD}_{2} R(x)=0$ everywhere. In a letter, mailed to Cantor from Zurich, Schwarz supplied the second step in the proof: a continuous function $F$ with $\mathrm{SD}_{2} F(x)=0$ everywhere must be linear. Finally then one has a relation 


$$
R(x)=\alpha x+\beta=a_{0} x^{2} / 4-\sum_{n=1}^{\infty}\left(a_{n} \cos n x+b_{n} \sin n x\right) / n^{2}
$$

Simple arguments show that this can hold only if all the coefficients vanish.

Our interests here are mostly in the property of the second order symmetric derivative expressed by this theorem of Schwarz which we now prove. The proof uses recognizably nineteenth century methods.

Theorem 1.12 (Schwarz) Let $F$ be continuous on the interval $[a, b]$ and suppose that $S D_{2} F(x)=0$ at every point of $(a, b)$. Then $F$ is linear in this interval.

Proof. Let $\epsilon>0$ and define the functions

$$
G(x)=F(x)-F(a)-\frac{F(b)-F(a)}{b-a}(x-a)+\epsilon(x-a)(x-b)
$$

and

$$
H(x)=F(x)-F(a)-\frac{F(b)-F(a)}{b-a}(x-a)-\epsilon(x-a)(x-b) .
$$

We prove that $G(x) \leq 0$ everywhere in $[a, b]$. If not then, because $G(a)=G(b)=$ 0 , there is a point $c \in(a, b)$ at which a positive maximum is attained. At such a point the derivative $\mathrm{SD}_{2} G(c)$ cannot be positive and yet this would contradict the fact that $\mathrm{SD}_{2} F(c) \geq 0$. An identical proof establishes that $H(x) \geq 0$ everywhere in $[a, b]$.

This gives

$$
\left|F(x)-F(a)-\frac{F(b)-F(a)}{b-a}(x-a)\right| \leq \epsilon(b-a)^{2}
$$

for all $\epsilon>0$. From this the linearity of $F$ evidently must follow.

Later researchers extended this theorem to provide a more delicate analysis. This variant is apparently due to de la Vallée Poussin.

Theorem 1.13 Let $F$ be a continuous function on the interval $[a, b]$. Suppose that $F$ satisfies the condition

$$
\limsup _{t \rightarrow 0}\left|\frac{F(x+t)+F(x-t)-2 F(x)}{t^{2}}\right|<+\infty
$$

at every point of $(a, b)$ and suppose that $S D_{2} F(x)=0$ at almost every point of $(a, b)$. Then $F$ is linear in this interval.

Proof. The proof uses a standard device from the early years of this century to push the argument used in the proof of Theorem 1.12 to handle the exceptional 
set of measure zero. We just insert the following addition into the proof of that theorem. Let $E$ be the set of points $x$ at which $\mathrm{SD}_{2} F(x)=0$ may fail. As $E$ has measure zero there is a continuous increasing function $k(x)$ on $[a, b]$ whose derivative on $E$ is infinite and such that $0=k(a)<k(b)<\epsilon$. We may choose $k$ so as to have a positive and finite or infinite derivative everywhere (see $[18$, p. 229]). Let $K(x)=\int_{a}^{x} k(x) d x$ and in the defining equation (1.9) for $G(x)$ add on the extra term $K(x)$.

Now

$$
\mathrm{SD}_{2} G(x) \geq \mathrm{SD}_{2} F(x)+2 \epsilon+\mathrm{SD}_{2} K(x)
$$

at every point $x$. But $\mathrm{SD}_{2} K(x)$ is everywhere positive and $\underline{\mathrm{SD}}_{2} K(x)=+\infty$ at every point $x$ with $k^{\prime}(x)=+\infty$. Thus we can conclude, as before, that $\underline{\mathrm{SD}}_{2} G(x)>0$ at every point. The argument continues more or less unchanged. The same addendum is, of course, necessary to handle the function $H$ and so the proof, in its modified form, works for this variant of Theorem 1.12.

There are further directions that this theorem of Schwarz can take us and that we will explore in greater depth in later chapters. Here we are content to state the results without proof. The first theorem asserts the Schwarz theorem in the context of a convexity result; if $f$ and $-f$ are both convex then $f$ is linear and so Theorem 1.12 above follows directly from this version. In Chapter 5 a complete account of convexity theorems can be found.

Theorem 1.14 Let $F$ be continuous on the interval $[a, b]$ and suppose that that $S D_{2} F(x) \geq 0$ at every point of $(a, b)$. Then $F$ is convex in this interval.

Yet another way of looking at the Schwarz theorem is due to de la Vallée Poussin. Note that Theorem 1.12 follows directly from this version too. These ideas will be explored and generalized in Chapter 9 .

Theorem 1.15 Let $F$ be continuous on the interval $[a, b]$ and suppose that that $S D_{2} F(x)=g(x)$ exists at every point of $(a, b)$. If $g$ is Lebesgue integrable on $(a, b)$ then

$$
F(x)=\int_{a}^{x} \int_{a}^{t_{1}} g\left(t_{2}\right) d t_{2} d t_{1}+c_{0}+c_{1} x
$$

where $c_{0}$ and $c_{1}$ are constants.

\subsection{Auerbach Theorem}

We return now to the first order symmetric derivative, but armed with some of the tools obtained in our study of the second symmetric derivative. We know already something about a function with an everywhere vanishing symmetric derivative. Here is Auerbach's generalized version of this with its original 1926 
proof. In Chapter 2 we will discover that a function that satisfies the condition (1.11) on an interval is continuous off a countable set and so the conclusion of the theorem can be considerably strengthened.

Theorem 1.16 (Auerbach) Let $f$ be a real valued measurable function on the interval $[a, b]$ that satisfies the condition

$$
\limsup _{t \rightarrow 0}\left|\frac{f(x+t)-f(x-t)}{2 t}\right|<+\infty
$$

at every point of $(a, b)$ and suppose that $S D f(x)=0$ at almost every point of $(a, b)$. Then $f$ is almost everywhere constant in this interval.

Proof. If $f$ is integrable then this theorem is almost a direct corollary of Theorem 1.13. Simply take $F$ as an indefinite integral of $f$ and then the conditions of the present theorem translate exactly into the conditions of that theorem. We see that

$$
\begin{aligned}
& \underset{h \rightarrow 0}{\limsup }\left|\frac{F(x+h)+F(x-h)-2 F(x)}{h^{2}}\right| \\
& \quad=\limsup _{h \rightarrow 0}\left|\frac{1}{h^{2}} \int_{0}^{h} \frac{f(x+t)-f(x-t)}{2 t} 2 t d t\right| \\
& \leq \limsup _{h \rightarrow 0}\left|\frac{f(x+h)-f(x-h)}{2 h}\right| .
\end{aligned}
$$

Thus the condition (1.11) for $f$ is exactly the condition (1.10) for $F$. Also, since we assume that $\operatorname{SD} f(x)=0$ at almost every point of $(a, b)$, it follows that $\mathrm{SD}_{2} F(x)=0$ at almost every point of $(a, b)$. Theorem 1.13 now shows that $F$ is linear and so $F^{\prime}=f$ is almost everywhere constant.

Accordingly what we need to do is show how to extend the result from integrable functions to arbitrary measurable ones. Define the functions

$$
f_{n}(x)=\frac{|f(x)+n|-|f(x)-n|}{2} .
$$

These are measurable and bounded and so integrable. Moreover a simple computation shows that the symmetric difference quotient for the $f_{n}$ is easily computed in terms of that for $f$ :

$$
\begin{aligned}
& \left|\frac{f_{n}(x+h)-f_{n}(x-h)}{2 h}\right| \\
& \quad=\left|\frac{|f(x+h)+n|-|f(x-h)+n|}{4 h}-\frac{|f(x+h)-n|-|f(x-h)-n|}{4 h}\right| \\
& \quad \leq\left|\frac{|f(x+h)+n|-|f(x-h)+n|}{4 h}\right|+\left|\frac{|f(x+h)-n|-|f(x-h)-n|}{4 h}\right| \\
& \quad \leq\left|\frac{f(x+h)-f(h-h)}{2 h}\right| .
\end{aligned}
$$


Now from what we have already proved we know that each $f_{n}$ is almost everywhere constant, say $c_{n}$, outside of a set $N_{n}$ of measure zero. Since $f(x)=$ $\lim _{n \rightarrow \infty} f_{n}(x)$ this means that $f(x)=\lim _{n \rightarrow \infty} c_{n}$ outside of $N=\bigcup_{n=1}^{\infty} N_{n}$. Thus $f$ is constant off the measure zero set $N$ and the proof is complete.

\subsection{Borel Symmetric Derivative}

Borel defined a variant on the ordinary derivative that he called a mean-value derivative (derivée moyenne); for an integrable function $f$ the derivative is computed using the quotient

$$
\frac{1}{h} \int_{0}^{h} \frac{f(x+t)-f(x)}{t} d t
$$

A symmetric version of this has some utility too and has been introduced by Khintchine [151, p. 220]. We present the definitions and prove the main observation of Khintchine, that this derivative is intimately linked to the second symmetric derivative. The proof is exactly the original one from [151, pp. 220221]; a more detailed set of relations between these two derivation processes will appear in Section 7.5 much later.

The symmetric-Borel derivates of an integrable $f$ at a point $x$ are defined as

$$
\overline{\mathrm{SBD}} f(x)=\limsup _{h \rightarrow 0} \frac{1}{h}\left\{\int_{0}^{h} \frac{f(x+t)-f(x-t)}{2 t} d t\right\}
$$

and

$$
\underline{\operatorname{SBD}} f(x)=\liminf _{h \rightarrow 0} \frac{1}{h}\left\{\int_{0}^{h} \frac{f(x+t)-f(x-t)}{2 t} d t\right\} \text {. }
$$

If the two extreme derivates are equal and finite then $f$ is said to have a symmetric Borel derivative at the point $x$ and the common value is denoted as SBD $f(x)$. Here the integral may be taken as an improper Lebesgue integral, i.e. interpreted in the sense that $f$ is integrable in a neighbourhood of the point $x$ and $\int_{0}^{h}$ is taken to mean $\lim _{\epsilon \rightarrow 0+} \int_{c}^{h}$. (Later on the Denjoy-Perron integral will be used in this context.)

Theorem 1.17 (Khintchine) Let $f$ be integrable in a neighbourhood of a point $x$ and let $F$ be an indefinite integral of $f$. If the second symmetric derivative of $F$ exists at a point $x$, then the symmetric Borel derivative of $f$ exists there too and

$$
S D_{2} F(x)=S B D f(x)
$$

Proof. The proof is obtained from the elementary differentiation identity 


$$
\begin{aligned}
& \frac{d}{d h}\left\{\frac{F(x+h)+F(x-h)-2 F(x)}{h}\right\} \\
& =\frac{f(x+h)-f(x-h)}{h}-\frac{F(x+h)+F(x-h)-2 F(x)}{h^{2}}
\end{aligned}
$$

which must hold almost everywhere in some interval $(0, \delta)$. We integrate this between the limits $p$ and $q$ for any $0<p<q<\delta$ and divide by $q$ to obtain

$$
\begin{gathered}
\frac{F(x+q)+F(x-q)-2 F(x)}{q^{2}}-\frac{F(x+p)+F(x-p)-2 F(x)}{p q}= \\
\frac{1}{q} \int_{p}^{q} \frac{f(x+t)-f(x-t)}{t} d t-\frac{1}{q} \int_{p}^{q} \frac{F(x+t)+F(x-t)-2 F(x)}{t^{2}} d t .
\end{gathered}
$$

The hypotheses of the theorem require that

$$
\frac{F(x+h)+F(x-h)-2 F(x)}{h^{2}} \rightarrow c
$$

for some $c$ as $h \rightarrow 0$. Now we let first $p \rightarrow 0$ and then $q \rightarrow 0$ in this identity. The two fractions on the left are easily handled and the second integral on the right requires only an application of the first mean value theorem for integrals. Thus we obtain

$$
c=\lim _{q \rightarrow 0} \frac{1}{q} \int_{p}^{q} \frac{f(x+t)-f(x-t)}{t} d t-c
$$

and hence

$$
\lim _{q \rightarrow 0} \frac{1}{q} \int_{p}^{q} \frac{f(x+t)-f(x-t)}{2 t} d t=c
$$

exactly as required, completing the proof.

As an application of Theorem 1.17 we can produce a variant on the theorems of Section 1.4. The function

$$
L(x)=a_{0} x / 2+\sum_{n=1}^{\infty}\left(a_{n} \sin n x-b_{n} \cos n x\right) / n,
$$

obtained by formally integrating the trigonometric series (1.1) once, is called the Lebesgue function for the series. If the coefficients $a_{n}, b_{n} \rightarrow 0$ then this series is known to converge almost everywhere to a Lebesgue integrable function. As a result of Theorem 1.17 the following is just a rewording of Riemann's first theorem (Theorem 1.9).

Theorem 1.18 Let $L$ be the Lebesgue function for the trigonometric series (1.5) with $a_{n}, b_{n} \rightarrow 0$. If (1.5) converges to a finite sum $s$ at a point $x_{0}$, then $S B D L\left(x_{0}\right)=s$. 


\subsection{Approximate Symmetric Derivative}

To complete the picture suggested by Theorem 1.18 we point out that yet another symmetric derivative may be used in place of the Borel symmetric derivative in this application to convergent trigonometric series. This allows us to introduce at an early stage a concept that will play an important role in the sequel.

The symmetric derivative can be generalized by using an approximate limit in place of the ordinary limit. This, in particular, allows for the differentiation of a function defined merely almost everywhere (as was the case for the Borel symmetric derivative). For any measurable function $f$ defined almost everywhere in a neighbourhood of a point $x_{0}$, we write

$$
\operatorname{ASD} f\left(x_{0}\right)=\operatorname{app} \lim _{t \rightarrow 0} \frac{f\left(x_{0}+t\right)-f\left(x_{0}-t\right)}{2 t}
$$

employing an approximate limit, and refer to this as the approximate symmetric derivative of $f$. When the ordinary symmetric derivative exists so too does the approximate symmetric derivative, but not conversely of course. The study of the approximate symmetric properties of the Lebesgue function of trigonometric series was initiated by Rajchman and Zygmund; this material can be found in [313, Chap. IX, §2]. The methods are very much rooted in the study of trigonometric series and will not be reproduced here. The assumption on the coefficients must be made only in order that the Lebesgue function exist almost everywhere; as Zygmund remarks, the assumption

$$
a_{n}, b_{n}=O\left(n^{\frac{1}{2}-\epsilon}\right) \quad(\epsilon>0)
$$

would be enough.

Theorem 1.19 (Rajchman-Zygmund) Let $L$ be the Lebesgue function for the trigonometric series (1.5) with $a_{n}, b_{n} \rightarrow 0$. If (1.5) converges to a finite sum $s$ at a point $x_{0}$, then $A S D L\left(x_{0}\right)=s$.

Proof. This is proved in Zygmund [313, Vol. I, Theorem 2.22, p. 324].

This theorem seems to put us in position to offer a different proof of the Cantor uniqueness theorem mentioned in Section 1.5. We wish to show that, if a trigonometric series

$$
a_{0} / 2+\sum_{n=1}^{\infty}\left(a_{n} \cos n x+b_{n} \sin n x\right)
$$

converges everywhere to 0 , then the coefficients $a_{n}, b_{n}=0$. Take the Lebesgue function $L$ for the series; by the Rajchman-Zygmund theorem $\operatorname{ASD} L(x)=0$ 
everywhere. It should follow that $L$ is constant (almost everywhere constant that is) and it is straightforward then to show that the coefficients vanish as required. But, surprisingly, it is no easy matter to prove that a measurable function $L$ for which $\operatorname{ASD} L(x)=0$ everywhere is equivalent to a constant function. The techniques for proving this were not developed until 1988. A full discussion will appear in Chapter 5 .

\subsection{Higher Order Symmetric Derivatives}

The first and second symmetric derivatives can also be viewed as the initial steps in a hierarchy of symmetric derivatives. We shall not have too much to say in the sequel about these higher order derivatives but we can present the basic facts here.

The method of the elementary calculus whereby higher order derivatives are introduced merely by iterating the derivation process is not of much use in many parts of real analysis. The existence of $f^{\prime \prime}\left(x_{0}\right)$ requires the existence of $f^{\prime}(x)$ for all $x$ in a neighbourhood of $x_{0}$ and this is a severe restriction. The method proposed by Peano in 1891 avoids this by modeling higher order derivation on Taylor's Theorem.

Definition 1.20 Let $k \in \mathbb{N}$. A function $f$ is said to have a $k$-th order Peano derivative at a point $x_{0}$ if

$$
f\left(x_{0}+h\right)=P(h)+o\left(h^{k}\right) \quad(\text { as } h \rightarrow 0)
$$

where $P$ is a polynomial of degree $k$ or less. In this case we write

$$
\operatorname{PD}^{(k)} f\left(x_{0}\right)=P^{(k)}(0) \text {. }
$$

Of course the first order Peano derivative is easily seen to be merely the ordinary derivative and $\operatorname{PD}^{(1)} f\left(x_{0}\right)=f^{\prime}\left(x_{0}\right)$. For higher orders, $k=2,3 \ldots$, simple examples illustrate that the concept is much more general than any iterated scheme of differentiation. For a survey of material on this derivative see Evans and Weil [98] and the continuation of this survey in Weil [305].

It is entirely natural to develop symmetric versions of the Peano derivative. This was initiated by Ch. de la Vallée Poussin in 1908 who also established their role in certain problems arising from summability and differentiation questions in trigonometric series. We report on some of these ideas here; all this material may be found in [313, Vol. II, Ch. XI] which the reader should consult for proofs and further refinements.

Definition 1.21 Let $k \in \mathbb{N}$. A function $f$ is said to have a $k$-th order symmetric Peano derivative at a point $x_{0}$ if 


$$
\frac{f\left(x_{0}+h\right)+(-1)^{k} f\left(x_{0}-h\right)}{2}=P(h)+o\left(h^{k}\right) \quad(\text { as } h \rightarrow 0)
$$

where $P$ is a polynomial of degree $k$ or less. In this case we write

$$
\mathrm{SD}^{(k)} f\left(x_{0}\right)=P^{(k)}(0)
$$

There are some immediate observations. One checks that the first two cases $(k=1,2)$ give exactly the same derivatives as before, justifying our use of this notation in this case. The expression in (1.13) employs the even part of $f$ for $k$ even and the odd part of $f$ for $k$ odd. Thus these derivatives can be considered as arising from the symmetric structure of $f$ : the even order derivatives represent even properties of the function and the odd order derivatives represent odd properties. The polynomial $P$ appearing in (1.13) can contain only even or odd terms depending on whether $k$ is even or odd. Thus, while the existence of the derivative $\mathrm{SD}^{(k)} f\left(x_{0}\right)$ will require also the existence of $\operatorname{SD}^{(k-2)} f\left(x_{0}\right)$, $\mathrm{SD}^{(k-4)} f\left(x_{0}\right) \ldots$, there is no necessity for $\mathrm{SD}^{(k-1)} f\left(x_{0}\right)$ to exist. Again this just reflects the even/odd nature of these derivatives.

The symmetric derivatives play an important role in the study of trigonometric series. We report on just one aspect. Note the similarity between the following theorem and Theorem 1.9, Theorem 1.18 and and Theorem 1.19.

Theorem 1.22 Let $k \in I N, \alpha \in I R$ with $k>\alpha+1>0$. Suppose that the trigonometric series (1.5) is summable $(C, \alpha)$ at $x_{0}$ to a finite sum $s_{0}$. Suppose that the series (1.5) integrated term by term $k$ times converges ${ }^{1}$ in a neigbourhood of $x_{0}$ to a sum $f(x)$. Then the $k$-th order symmetric derivative of $f$ exists at $x_{0}$ and $\mathrm{SD}^{(k)} f\left(x_{0}\right)=s_{0}$.

Proof. This is proved in Zygmund [313, Vol. II, Theorem 2.1, p. 66].

In place of the definitions used here one might instead employ the higher order differences of Section 1.2.3. Thus it is reasonable to define

$$
\mathrm{SD}^{(k)} f\left(x_{0}\right)=\lim _{t \rightarrow 0} \frac{\Delta_{s}^{k} f\left(x_{0}, t\right)}{t^{k}}
$$

as a $k$-th order symmetric derivative. In the literature these are called Riemann's derivatives. In fact if $\mathrm{SD}^{(k)} f\left(x_{0}\right)$ exists in the sense of Definition 1.21 then it exists in the sense of (1.15). The cases $k=1,2$ are of course the same for both definitions.

We shall not pursue these ideas, however, as they do not represent symmetric properties in the narrow sense we use here.

\footnotetext{
${ }^{1}$ This is certainly the case if $\left|a_{n}\right|+\left|b_{n}\right|=o\left(n^{\alpha}\right)$.
} 


\subsection{Khintchine Theorem}

Thus far our methods have been mostly of a classical nature and have not required an analysis that uses any subtle geometric features of the symmetric derivative. We turn now to what must be considered a fundamental property of symmetric derivates, one that has been a central theorem in the history of the subject and a focus for much generalization. This theorem shows that in the most frequent situations in analysis, those in which sets of measure zero may be ignored or play no role, the symmetric derivative and the ordinary derivative cannot be distinguished.

We reproduce the original proof of Khintchine from [151, p. 217] with commentary and some notational changes.

Theorem 1.23 (Khintchine) Let $f$ be a measurable function. Then $f$ is differentiable at almost every point $x$ at which

$$
\limsup _{h \rightarrow 0} \frac{f(x+h)-f(x-h)}{2 h}<+\infty .
$$

Proof. Let $E$ denote the set of points $x$ at which the inequality (1.16) holds. The first step in the proof invokes a standard device in derivation theory and one which we will repeat many times throughout this work. The relation (1.16) asserts a strictly local property; expressed in other words it asserts that at every point $x$ in the set $E$ there are positive numbers $\delta(x)$ and $M(x)$ so that

$$
x \in E, 0<h<\delta(x) \Longrightarrow \frac{f(x+h)-f(x-h)}{2 h}<M(x) .
$$

But such a local property does not allow for any argument about the existence of the derivative. Instead we would require such a statement to hold uniformly. The device we use is to partition the set $E$ in such a way that this implication does indeed hold uniformly on each member of the partition.

Lemma 1.24 If the limit

$$
\limsup _{h \rightarrow 0} \frac{f(x+h)-f(x-h)}{2 h}<+\infty
$$

holds at every point of a set $E$ then there is a partition $\left\{E_{n}\right\}$ of the set $E$ and a sequence of positive numbers $\left\{M_{n}\right\}$ so that

$$
x \in E_{n}, 0<h<1 / n \Longrightarrow \frac{f(x+h)-f(x-h)}{2 h}<M_{n} .
$$

To prove Lemma 1.24 it is enough to define the sequence of sets 


$$
A_{n}=\{x \in E: \delta(x)<1 / n, M(x)<n\}
$$

and then to define $M_{n}=n, E_{1}=A_{1}$ and, for $n>1$,

$$
E_{n}=A_{n} \backslash \bigcup_{k<n} A_{k} .
$$

The second step in Khintchine's proof is to obtain, from Lemma 1.24, estimates on the size of the Dini derivatives at points in the sets $E_{n}$. We use the notation $\underline{\mathrm{D}}^{+} f(x), \underline{\mathrm{D}}^{-} f(x), \overline{\mathrm{D}}^{+} f(x)$ and $\overline{\mathrm{D}}^{-} f(x)$ for the four Dini derivatives of a function $f$ at a point $x$. The best we can hope for here is to work with density points of these sets, but since almost every point is a point of (exterior) density this will be enough for the conclusion of the theorem. Let us state this step as a separate lemma.

Lemma 1.25 Suppose that $f$ is measurable and, at every point $x$ in a set $E$, that

$$
0<h<\delta \Longrightarrow \frac{f(x+h)-f(x-h)}{2 h}<M .
$$

Then at every point $x_{0}$ of exterior density of $E$ the estimate $\bar{D} f\left(x_{0}\right) \leq M$ must hold.

To prove Lemma 1.25 we first simplify the computations by replacing $g(x)=$ $f(x)-M x$ so that the hypothesis now reads

$$
x \in E, 0<h<\delta \Longrightarrow \frac{g(x+h)-g(x-h)}{2 h}<0
$$

and the conclusion required is that at every point $x_{0}$ of exterior density of $E$ we have $\overline{\mathrm{D}} g\left(x_{0}\right) \leq 0$. It is enough to argue that $\overline{\mathrm{D}}^{+} g\left(x_{0}\right) \leq 0$ and then, by identical arguments, $\overline{\mathrm{D}}^{-} g\left(x_{0}\right) \leq 0$ must follow too.

If $x_{0}$ is a point of density of $E$ then we may choose a positive number $\delta_{1}<\delta$ so that

$$
\left|E \cap\left(x_{0}, x_{0}+t\right)\right|>3 t / 4
$$

if $0<t<\delta_{1}$. The proof is completed now merely by showing that $g(x) \leq g\left(x_{0}\right)$ for all $x_{0}<x<x_{0}+\delta_{1}$ for then the inequality $\overline{\mathrm{D}}^{+} g(x) \leq 0$ must follow. This in turn may be proved by proving the impossibility of a point $x_{1}$ in this interval for which $g\left(x_{0}\right)<g\left(x_{1}\right)$. The remainder of the proof is devoted to obtaining a contradiction in this situation.

Define the two sets

$$
A=\left\{x \in\left(x_{0}, x_{1}\right): g\left(x_{0}\right)<g(x)\right\}
$$

and

$$
B=\left\{x \in\left(x_{0}, x_{1}\right): g(x)<g\left(x_{1}\right)\right\}
$$


Because $g\left(x_{0}\right)<g\left(x_{1}\right)$ these sets exhaust the interval $\left(x_{0}, x_{1}\right)$ and because $f$, and hence $g$, is measurable both $A$ and $B$ are measurable so that one of these sets has measure exceeding $\left(x_{1}-x_{0}\right) / 2$.

If $A$ has measure exceeding $\left(x_{1}-x_{0}\right) / 2$ then the set $A^{\prime}=\frac{1}{2}\left(x_{0}+A\right)$ is contained in $\left(x_{0},\left(x_{0}+x_{1}\right) / 2\right)$ and has measure at least $\left(x_{1}-x_{0}\right) / 4$. This combined with (1.17) shows that the intersection $E \cap A^{\prime} \cap\left(x_{0}, x_{1}\right)$ cannot be empty. Let $z$ be a point in this intersection. Write $h=z-x_{0}$ and note that $0<h<\delta$. Then $z-h=x_{0}, z \in E$ and $z+h \in A$ and we must have

$$
g(z+h)-g(z-h)<0
$$

and

$$
g(z-h)=g\left(x_{0}\right)<g(z+h)
$$

which is the desired contradiction.

On the other hand if $B$ has measure exceeding $\left(x_{1}-x_{0}\right) / 2$ then the set $B^{\prime}=$ $\frac{1}{2}\left(x_{1}+B\right)$ is contained in $\left(\left(x_{0}+x_{1}\right) / 2, x_{1}\right)$ and has measure at least $\left(x_{1}-x_{0}\right) / 4$. This combined again with (1.17) shows that the intersection $E \cap B^{\prime} \cap\left(x_{0}, x_{1}\right)$ cannot be empty. If $z$ is a point in this intersection then, with $h=x_{1}-z$, $0<h<\delta, z+h=x_{1}, z \in E$ and $z-h \in B$ we must have

$$
g(z+h)-g(z-h)<0
$$

and

$$
g(z+h)=g\left(x_{1}\right)>g(z-h)
$$

which is again a contradiction. Thus in both cases we obtain a contradiction and Lemma 1.25 is proved.

Combining Lemmas 1.24 and 1.25 we can now prove Theorem 1.23. Together these facts and the fact that almost every point of any set is a point of exterior density of that set show that at almost every point $x \in E$ the derivate $\overline{\mathrm{D}} f(x)$ is finite. But it is well known in the theory of the ordinary derivative that, at almost every such point, the function $f$ must have a finite derivative (see [251, Theorems 7.2 and 10.1]). This completes the proof.

As an immediate corollary we conclude that, at least for measurable functions and in situations where sets of measure zero may be ignored, the symmetric derivative is not distinct from the ordinary derivative. In the statement of this corollary note that infinite symmetric derivatives would be allowed.

Corollary 1.26 (Khintchine) If a measurable function $f$ has a symmetric derivative at almost every point then $f$ is almost everywhere differentiable in the ordinary sense. 


\section{References}

[1] J. M. Anderson and L. D. Pitt. Probabilistic behaviour of functions in the Zygmund spaces $A^{*}$ and $\lambda^{*}$. Proc. London Math. Soc. (3), 59: 558-592, 1989.

[2] T. Anghelutza. Sur une propriété des polynômes. Bull. Sci. Math. (2), 63:239246, 1939.

[3] J. Ash, J. Cohen, C. Freiling, L. Gluck, E. Rieders, and G. Wang. Characterizations and generalizations of continuity. Proc. Amer. Math. Soc., (to appear) 1993.

[4] J. M. Ash. Generalizations of the Riemann derivative. Trans. American Math. Soc., 126:181-191, 1967.

[5] J. M. Ash. A new proof of uniqueness for [multiple] trigonometric series. Proc. American Math. Soc., 107:409-410, 1989.

[6] J. M. Ash. A new, harder proof that continuous functions with Schwarz derivative 0 are lines. Contemporary Aspects of Fourier Analysis, Bray and Stanojevi'vc eds., 1993.

[7] J. M. Ash, J. Cohen, C. Freiling, and D. Rinne. Generalizations of the wave equation. Trans. Amer. Math. Soc., 337: 1993.

[8] J. M. Ash, C. Freiling, and D. Rinne. Uniqueness of rectangularly convergent trigonometric series. Annals of Math., 137:145-166, 1993.

[9] H. Auerbach. Sur les dérivées generalisées. Fund. Math., 8:49-55, 1926.

[10] C. E. Aull. The first symmetric derivative. American Math. Monthly, 74:708$711,1967$.

[11] N. Bary. A treatise on trigonometric series, Vol. I and II. Pergamon Press, New York, 1964.

[12] C. L. Belna. Symmetric continuity of real functions. Prac. American Math. Soc., 87:99-102, 1983.

[13] C. L. Belna, M. J. Evans, and P. Humke. Symmetric and ordinary differentiation. Proc. American Math. Soc., 72:261-267, 1978.

[14] C. L. Belna, M. J. Evans, and P. Humke. Symmetric and strong differentiation. American Math. Monthly, 86:121-123, 1979.

[15] F. Berstein and G. Doetsch. Zur Theorie der konvexen Funktionen. Math. Ann., $76: 514-526,1915$.

[16] H. Blumberg. On convex functions. Trans. American Math. Soc., 29:40-44, 1919.

[17] M. W. Botsko. A unified treatment of various theorems in elementary analysis. Amer. Math. Monthly, 94:450-452, 1987. 
[18] A. M. Bruckner. Differentiation of Real Functions. Lecture Notes in Mathematics 659. Springer-Verlag, Berlin, 1978.

[19] A. M. Bruckner, J. Ceder, and M. Weiss. On uniform limits of Darboux functions. Colloq. Math., 45:65-77, 1966.

[20] Yu. A. Brudnyi. On the maximum modulus of a quasi-smooth function. Uspehi Mat. Nauk (N.S.), 12:273-275, 1957.

[21] Z. Buczolich. Convexity and symmetric derivates of measurable functions. Real Anal. Exchange, 16:187-196, 1990/91.

[22] Z. Buczolich, M. J. Evans, and P. D. Humke. Approximate high order smoothness. Acta Math. Hung., 61 (3-4), 369-388 (1993).

[23] Z. Buczolich and M. Laczkovich. Concentrated Borel measures. Acta. Math. Hungar., 57:349-362, 1991.

[24] P. S. Bullen. Construction of primitives of generalized derivatives with applications to trigonometric series. Canad. J. Math., 13:48-58, 1961.

[25] P. S. Bullen. Nonabsolute integrals: a survey. Real Anal. Exchange, 5:195-259, 1979.

[26] P. S. Bullen. Denjoy's index and porosity. Real Anal. Exchange, 10:85-144, 1984.

[27] P. S. Bullen and C. M. Lee. On the integrals of Perron type. Trans. American Math. Soc., 182:481-501, 1973.

[28] P. S. Bullen and C. M. Lee. The $\mathrm{SC}_{n} \mathrm{P}-$ integral and the $\mathrm{P}^{n+1}$-integral. Canad. J. Math., 25:1274-1284, 1973.

[29] P. S. Bullen and S. N. Mukhopadhyay. Integration by parts formulae for some trigonometric integrals. Proc. London Math. Soc., (3) 29:159-173, 1974.

[30] P. S. Bullen and S. N. Mukhopadhyay. Generalized continuity of higher order symmetric derivatives. Southeast Asian Bull. Math., 13:127-137, 1989.

[31] P. S. Bullen and S. N. Mukhopadhyay. The integrability of Riemann summable trigonometric series. Canad. Math. Bull., 33:273-281, 1990.

[32] H. Burkill. A note on trigonometric series. J. Math. Anal. Appl, 40:39-44, 1972.

[33] H. Burkill. Fourier series of SCP-integrable functions. J. Math. Anal. Appl., $57: 587-609,1977$.

[34] J. C. Burkill. The expresssion of trigonometric series in Fourier form. J. London Math. Soc, 11:43-48, 1936.

[35] J. C. Burkill. Integrals and trigonometric series. Proc. London Math. Soc., (3) $1: 46-57,1951$.

[36] J. C. Burkill. Corrigendum: Integrals and trigonometric series. Proc. London Math. Soc., (3) 47:192, 1983.

[37] P. Butzer. Bezeihungen zwischen den Riemannscen, Taylorscen und gewohnlichen reelwertiger Funktionen. Math. Ann., 144:275-298, 1961.

[38] P. Butzer and W. Kozakiewicz. On the Riemann derivatives for integrable functions. Canad. J. Math., 6:572-581, 1954.

[39] A. Calderon and A. Zygmund. Local properties of solutions of elliptic partial differential equations. Studia Math., 20:171-225, 1961.

[40] G. Cantor. Über unendliche, lineare Punktmannigfaltigkeiten. Math. Ann., $23: 453-488,1884$.

[41] G. Cantor. Gessamelte Abhandlungen. Georg Olms, Hilldesheim, 1962. 
[42] Z. Charzyński. Sur les fonctions dont la derivée symetrique est partout finie. Fund. Math., 21:214-225, 1931.

[43] M. Chlebík. On symmetrically continuous functions. Real Anal. Exchange, 13:34, $1987 / 88$.

[44] K. Ciesielski and L. Larson. Uniformly antisymmetric functions. Real Anal. Exchange, 19(1), 1993/94.

[45] E. Corominas. Dérivation de Riemann-Schwarz. C. R. Acad. Sci. Paris Sér. I Math., 224:176-177, 1947.

[46] P. Cousin. Sur les fonctions de $n$ variables complexes. Acta Math., 19:1-61, 1895.

[47] G. E. Cross. The expression of trigonometric series in Fourier form. Canad. J. Math., 12:694-698, 1960.

[48] G. E. Cross. The relation between two definite integrals. Proc. American Math. Soc., 11:578-579, 1960.

[49] G. E. Cross. The relation between two symmetric integrals. Proc. American Math. Soc., 14:185-190, 1963.

[50] G. E. Cross. On the generality of the AP-integral. Canad. J. Math., 23:557-561, 1971.

[51] G. E. Cross. The $\mathrm{P}^{n}$-integral. Canad. Math. Bull., 18:493-497, 1975.

[52] G. E. Cross. Additivity of the $\mathrm{P}^{n}$-integral. Canad. J. Math., 30:783-796, 1978.

[53] G. E. Cross. The representation of $(\mathrm{C}, \mathrm{k})$-summable trigonometric series. Canad. Math. Bull., 21:149-158, 1978.

[54] G. E. Cross. The $\mathrm{SC}_{k+1} \mathrm{P}$-integral and trigonometric series. Proc. American Math. Soc, 69:297-302, 1978.

[55] G. E. Cross. Higher order Riemann complete integrals. Real Anal. Exchange, $11: 347-364,1985 / 86$.

[56] G. E. Cross and B. S. Thomson. Symmetric integrals and trigonometric series. Dissertationes Math. (Rozprawy Mat.), Vol. 319, 1992.

[57] U. B. Darji. Symmetric behavior in functions. Proc. American Math. Soc, November 1993.

[58] U. B. Darji. Symmetric functions, Lebesgue measurability and the Baire property. Proc. American Math. Soc., 118:1151-1158, 1993.

[59] U. B. Darji. Coanalytic sets and symmetric behavior in functions.

J. Math. Anal. Appl., (to appear).

[60] R. O. Davies. Symmetric sets are measurable. Real Anal. Exchange, 4:87-89, $1978 / 79$.

[61] R. O. Davies and F. Galvin. Solution to Query 5. Real Anal. Exchange, 2:74-75, 1976.

[62] S. De Sarkar and A. G. Das. Riemann derivatives and general integrals. Bull. Austral. Math. Soc., 35:187-211, 1987.

[63] M. Dehn Über die Zerlegung von Rechtecken in Rechtecke. Math. Ann., 57:314$332,1903$.

[64] A. Denjoy. Calcul de la primitive de la fonction dérivée la plus générale. $C . R$. Acad. Sci. Paris Sér. I Math., 154:1075-1078, 1912.

[65] A. Denjoy. Sur l'absolue convergence des séries trigonométrique. C. R. Acad. Sci. Paris Sér. I Math., 156:135-136, 1912. 
[66] A. Denjoy. Une extension de l'intégrale de M. Lebesgue. C. R. Acad. Sci. Paris Sér. I Math., 154:859-862, 1912.

[67] A. Denjoy. Calcul des coefficients d'une séries trigonométrique partout convergente. C. R. Acad. Sci. Paris Sér. I Math., 172:1218-1221, 1921.

[68] A. Denjoy. Leçons sur le calcul des coefficients d'une série trigonométrique. Hermann, Paris, 1941-49.

[69] A. Denjoy. Totalization des derivées premieres generalizés, 1. C. R. Acad. Sci. Paris Sér. I Math., 241:617-620, 1955.

[70] A. Denjoy. Totalization des derivées premieres generalizés, II. C. R. Acad. Sci. Paris Sér. I Math., 241:829-832, 1955.

[71] R. Dougherty and A. S. Kechris. The complexity of antidifferentiation. Advances in Math., 88:145-169, 1991.

[72] P. L. Dure, H. S. Shapiro, and A. L. Shields. Singular measures and domains not of Smirnov type. Duke Math. J., 33:247-254, 1966.

[73] W. L. Duren. Mathematical induction in sets. Amer. Math. Monthly, 64:19-21, 1957.

[74] T. K. Dutta. Generalized smooth functions. Acta. Math. Hungar., 40:29-37, 1982.

[75] T. K. Dutta. On uniform generalized symmetric derivatives. Indian J. Math., 33:63-74, 1991.

[76] T. K. Dutta and S. N. Mukhopadhyay. Generalized smooth functions. II. Acta. Math. Hungar., 55:47-56, 1990.

[77] A. F. Dzyadyk, V. K.Timan. On best approximation of quasi-smooth functions by ordinary polynomials. Doklady Akad. Nauk-SSSR (N.S.), 75:499-501, 1950.

[78] The Editors. Query 37. Real Anal. Exchange, 3:107, 1977/78.

[79] H. W. Ellis. On the relation between the $\mathrm{P}^{2}$-integral and the Cesàro-Perron scale of integrals. Trans. Roy. Soc. Canada (4), III (3) 46:29-32, 1952.

[80] P. Erdös. Some remarks on subgroups of real numbers. Colloq. Math., 42:119$120,1979$.

[81] M. Esser and O. Shisha. A modified differentiation. American Math. Monthly, 71:904-906, 1964.

[82] M. J. Evans. On continuous functions and approximate symmetric derivatives. Colloq. Math., 31:129-136, 1974.

[83] M. J. Evans. A symmetric condition for monotonicity. Bull. Math. Inst. Acad. Sinica, 6:85-91, 1978.

[84] M. J. Evans. Symmetric and smooth functions: a few questions and fewer answers. Real Anal. Exchange, 9:381-385, 1983/84.

[85] M. J. Evans. Peano differentiation and high order smoothness in $\mathrm{L}_{p}$. Bull. Inst. Math. Acad. Sinica, 13:197-209, 1985.

[86] M. J. Evans. Approximate smoothness of continuous functions. Colloq. Math., 54:307-313, 1987/88.

[87] M. J. Evans. Points of approximate continuity, approximate symmetry and Lpoints. Real Anal. Exchange, 13:25-27, 1987/88.

[88] M. J. Evans. A note on symmetric and ordinary differentiation. Real Anal. Exchange, 17:820-826, 1991. 
[89] M. J. Evans. Some theorems whose $\sigma$-porous exceptional sets are not $\sigma-$ symmetrically porous. Real Anal. Exchange, 17:809-814, 1991.

[90] M. J. Evans and S. Fu. Mean value properties for symmetrically differentiable functions. Real Anal. Exchange, 17:657-667, 1991.

[91] M. J. Evans and P. Humke. Approximate continuity points and L-points of integrable functions. Real Anal. Exchange, 11:390-410, 1985/86.

[92] M. J. Evans, P. Humke, and K. Saxe. A symmetric porosity conjecture of Zajícek. Real Anal. Exchange, 17:258-271, 1985/86.

[93] M. J. Evans and P. D. Humke. On equality of unilateral derivates. Proc. American Math. Soc., 79:609-613, 1980.

[94] M.-J. Evans and P.-D. Humke. A pathological approximately smooth function. Acta Math. Hungar., 46:211-215, 1985.

[95] M. J. Evans and L. Larson. Qualitative differentiation. Trans. American Math. Soc., 280:303-320, 1983.

[96] M. J. Evans and L. Larson. The continuity of symmetric and smooth functions. Acta. Math. Hungar., 43:251-257, 1984.

[97] M. J. Evans and R. W. Vallin Qualititative symmetric differentiation. Real Anal. Exchange 18:575-585 1992/93.

[98] M. J. Evans and C. E. Weil. Peano derivatives: a survey. Real Anal. Exchange, $7: 5-23,1981 / 82$.

[99] C. C. Ezzell and J. E. Nymann. An analogue of the Denjoy theorem for the symmetric derivative. Rev. Roumaine Math. Pures Appl., 17:237 - 241, 1972.

[100] H. Fejzić. Convex functions and Schwarz derivates. Proc. Amer. Math. Soc. (to appear).

[101] H. Fejzić and C. E. Weil. A repair of the proof of Ash-Marcinkiewicz-Zygmund theorem. Real Anal. Exchange (to appear).

[102] F. M. Filipczak. Sur la structure de l'ensemble des points oú une fonction n'admet pas de dérivée symétrique. Dissertationes Math. (Rozprawy Mat.), 130:1-49, 1975.

[103] F. M. Filipczak. Sur les dérivées symétriques des fonctions approximativement continues. Colloq. Math., 34:249-256, 1976.

[104] L. Filipczak. Exemple d'une fonction continue privé de dérivée symétrique partout. Colloq. Math., 20:249-253, 1969.

[105] J. Foran. Some relations of subgroups of the real numbers to Hausdorff measures. J. London Math. Soc., (2) 7:651-661, 1974.

[106] J. Foran. The symmetric and ordinary derivative. Real Anal. Exchange, 2:105$108,1977$.

[107] J. Foran and L. Larson. The symmetric derivation basis, approximate symmetric derivatives and montonicity. Real Anal. Exchange, 13:278-284, 1987/88.

[108] M. Foran. Symmetric functions. Real Anal. Exch., 1:38-40, 1976.

[109] L. R. Ford. Interval-additive propositions. Amer. Math. Monthly, 64:106-142, 1957.

[110] C. Freiling. A converse to a theorem of Sierpiński on almost symmetric sets. Real Anal. Exchange, 15:760-767, 1989/90.

[111] C. Freiling. Symmetric derivates, scattered and semi-scattered sets. Trans. American Math. Soc., 318:705-720, 1990. 
[112] C. Freiling and D. Rinne. A symmetric density property, monotonicity and the approximate symmetric derivative. Proc. American Math. Soc., 104:1098-1102, 1988.

[113] C. Freiling and D. Rinne. A symmetric density property for measurable sets. Real Anal. Exchange, 14:203-209, 1988-89.

[114] C. Freiling and D. Rinne. Symmetric derivates of non-measurable functions. Real Anal. Exchange, 14:517-522, 1988-89.

[115] C. Freiling and D. Rinne. An approximate analog of a theorem of Khintchine. Fund. Math., 135:55-59, 1990.

[116] C. Freiling, D. Rinne, and B. S. Thomson. A Riemann-type integral based on the second symmetric derivative. (manuscript), 1993.

[117] C. Freiling and B. S. Thomson. Scattered sets, chains and the Baire cateegory theorem. (manuscript), 1993.

[118] E. Freund. S-Null functions. Real Anal. Exchange, 16:319-321, 1990/91.

[119] H. Fried. Über die symmetrische Steigkeit von Funktionen. Fund. Math., 29:134-137, 1937.

[120] W. H. Gage and R. D. James. A generalised integral. Proc. Roy. Soc. Canada (4), 40:25-36, 1946 .

[121] K. Garg. A new notion of derivative. Real Analysis Exch., 75:65-84, 1981/82.

[122] N. Giovannelli. On a generalization of the $k$-pseudosymmetric derivative. Atti Accad. Sci. Lett. Arti Palermo Ser. (5), 38:219-229, 1978.

[123] C. Goffman. On Lebesgue's density theorem. Proc. Amer. Math. Soc., 1:384$388,1950$.

[124] C. Goffman and C. J. Neugebauer. On approximate derivatives. Proc. American Math. Soc., 11:962-966, 1960.

[125] E. Gorlich and R. Nessel. Uber Peano- und Riemann-Ableitungen in deer Norm. Arch. Math. (Basel), 18:399-410, 1967.

[126] E. Goursat. Sur la définition générale des fonctions analytiques d'après cauchy. Trans. Amer. Math. Soc., 1:14-16, 1900.

[127] G. Hamel. Eine Basis aller Zahlen und die unstetige Losungen der funktionalgleichung $f(x+y)=f(x)+f(y)$. Math. Ann., 60:459-462, 1905.

[128] L. Hammill. On sets of symmetric points. AMS Abstracts, 13:46, 1992.

[129] F. Hausdorff. Problème No. 62. Fund. Math., 25:578, 1935.

[130] R. Henstock. $N$-variation and $N$-variational integrals of set functions. Proc. London Math. Soc., (3) 11:109-133, 1961.

[131] R. Henstock. Linear Analysis. Butterworths, London, 1967.

[132] E. Hewitt and K. R. Stromberg. Some examples of nonmeasurable sets. $J$. Austrail. MAth. Soc., 18:236-238, 1974.

[133] E. W. Hobson. The theory of functions of a real variable and the theory of Fourier series I, II (2nd Ed.). Cambridge, Cambridge, 1926.

[134] P. D. Humke and M. Laczkovich. Monotonicity theorems for generalized Riemann derivatives. Rend. Circ. Mat. Palermo (2), 38:437-454, 1989.

[135] P. D. Humke and M. Laczkovich. An elementary proof of Freiling's symmetric covering lemma. Real Anal. Exchange, 16:549-551, 1990/91.

[136] P. D. Humke and M. Laczkovich. Parametric semicontinuity implies continuity. Real Anal. Exchange, 17:668-680, 1991/92. 
[137] P. D. Humke and T. Salát. Remarks on strong and symmetric differentiability of real functions. Acta Math. univ. Comenian., 52/53:235-241, 1987.

[138] R. D. James. A generalised integral (II). Canad. J. Math., 2:297-306, 1950.

[139] R. D. James. Integrals and summable trigonometric series. Bull. American Math. Soc., 61:1-15, 1955.

[140] J. Jaskula and B. Szkopińska. On the set of points of symmetric continuity. An. Univ. Bucureşti Mat., 37:29-35, 1988.

[141] J. M. Jedrzewski. Approximately smooth functions. Zeszyty Nauk. Uniw. Lodzk. Nauki Mat. Przyrod. Ser. II, 52:7-14, 1973.

[142] R. L. Jeffrey. Trigonometric series. Canad. Math. Congress Lecture Series (\#2), 1956.

[143] J. L. W. V. Jensen. Om konvekse funktioner og uligheder imellem middelvaerdier. Nyt. Tidsskrift for Mathematik, 16 B:49-69, 1905.

[144] F. John and L. Nirenberg. On functions of bounded mean oscillation. Comm. Pure Appl. Math., 14:415-426, 1961.

[145] F. B. Jones. Measure and other properties of a Hamel basis. Bull. Amer. Math. Soc. 48:472-481, 1942.

[146] G. Jungck. Interval induction. Amer. Math. Monthly, 73:295-297, 1966.

[147] J.-P. Kahane. Trois notes sur les ensembles parfaits linéaires. Enseignemant Math., 15:187-192, 1969.

[148] I. P. Kārkliņa. On quasi-smooth functions. Latvijas Valsts Univ. Zinatn. Raksti, 41:43-46, 1961.

[149] C. Kassimatis. Functions which have generalized Riemann derivatives. Canad. J. Math., 10:413-420, 1958.

[150] J. H. B. Kemperman. A general functional equation. Trans. American Math. Soc., 86:28-56, 1957.

[151] A. Khintchine. Recherches sur la structure des fonctions mesurables. Fund. Math., 9:212-279, 1927.

[152] P. Komjáth and S. Shelah. On uniformly antisymmetric functions. Real Anal. Exchange, 19(1), 1993/94.

[153] P. Kostyrko. On the symmetric derivative. Colloq. Math., 25:265-267, 1972.

[154] P. Kostyrko. On a local form of Jensen's functional equation. Aequationes Math., 30:65-69, 1986.

[155] P. Kostyrko. The symmetric derivative and the Darboux property. Real Anal. Exchange, 13:305-309, 1987/88.

[156] P. Kostyrko. Approximate symmetric derivatives are uniformly closed. Real Anal. Exchange, 17:652-656, 1991.

[157] P. Kostyrko. On locally symmetric and locally Jensen functions. Real Anal. Exchange, 17:405-408, 1991.

[158] P. Kostyrko, T. Neubrunn, Šalát T., and J. Smital. On locally symmetric and symmerically continuous functions. Real Anal. Exchange, 6:67-76, 1980.

[159] B. D. Kotljar. On quasi-smooth functions of two variables. Ukrain. Mat. Z, 16:383-385, 1964.

[160] Y. Kubota. An integral with basis and its application to trigonometric series. Bull. Fac. Sci. Ibaraki Univ. Ser. A, 5:1-8, 1973. 
[161] M. Kuczma. An introduction to the theory of functional equations and inequalities. Uniwersytet Ślaski, Warsaw, 1985.

[162] M. Kulbacka. Sur les ensembles stationaires et déterminants pour certaines classes de dérivées symétriques. Colloq. Math., 19:255-259, 1968.

[163] N. K. Kundu. On some conditions of symmetric derivatives implying monotonicity of functions. Rev. Roumaine Math. Pures Appl., 15:561-568, 1970.

[164] N. K. Kundu. On approximate symmetric derivative. Colloq. Math., 28:275$285,1973$.

[165] N. K. Kundu. On some properties of symmetric derivatives. Ann. Polon. Math., 30:9-18, 1974.

[166] K. Kuratowski. Topology. Academic Press, London, 1966.

[167] S. Kurepa. Convex functions. Glasnik Mat. Fiz. Astronom., (2) 11:89-93, 1956.

[168] J. Kurzweil and J. Jarník. On some extensions of the Perron integral on onedimensional intervals. An approach by integral sums fulfilling a symmetry condition. Funct. Approx. Comment. Math., 17:49-55, 1987.

[169] J. Kurzweil and J. Jarník. On a generalization of the Perron integral on onedimensional intervals. Ann. Polon. Math., 51:205-218, 1990.

[170] M. Laczkovich. A Bajre two function with non-Borel upper symmetric derivative. Real Anal. Exchange, 13:258-264, 1987/88.

[171] L. Larson. The Baire class of approximate symmetric derivates. Proc. American Math. Soc., 87:125-130, 1983.

[172] L. Larson. The symmetric derivative. Trans. American Math. Soc, 277:589$599,1983$.

[173] L. Larson. Symmetric real analysis: a survey. Real Anal. Exchange, 9:154-178, $1983 / 84$.

[174] L. Larson. The generalized Zahorski class structure of symmetric derivatives. Trans. American Math. Soc., 282:45-58, 1984.

[175] L. Larson. A method for showing generalized derivatives are in Baire class one. Classical real analysis, Series: Amer. Math. Soc., Providence, R.I., Contemp. Math., 42.:87-95, 1985.

[176] L. Larson. Approximate smoothness and Baire*1. Acta. Math. Hungar., 57:187$200,1991$.

[177] C. M. Lee. On the integrals of Perron type. Thesis, (U. B. C.), 1972.

[178] C. M. Lee. On integrals and summable trigonometric series. RAE, 4:66-68, 1978.

[179] C. M. Lee. Generalizations of Cesàro continuous functions and integrals of Perron type. Trans. Amer. Math. Soc., 266:461-481, 1981.

[180] C. M. Lee. A symmetric approximate Perron integral for the coefficient problem of convergent trigonometric series. Real Anal. Exchange, 16:329-339, 1990.

[181] N. Lusin. Sur l'absolue convergence des séries trigonométrique. C. R. Acad. Sci. Paris Sér. I Math., 155:580-582, 1912.

[182] N. Lusin. Sur une théorème fondamental du calcul intégral. Mat. Sbornik, $28: 266-294,1912$.

[183] W. A. J. Luxemburg. Problem 68. Niew Archief voor Wiskund, p. 121, 1965.

[184] N. G. Makarov. On the radial behaviour of Bloch functions. Soviet Math. Dokl., 40:505-508, 1990. 
[185] J. Marcinkiewicz. Sur les séries de Fourier. Fund. Math., 27:38-69, 1936.

[186] J. Marcinkiewicz and A. Zygmund. On the differentiability of functions and the summability of trigonometrical series. Fund. Math., 26:1-43, 1936.

[187] S. Marcus. Mulţimile $F_{\sigma}$ şi continuitatea simetrică. Bul. Şti. Acad. Rep. Pop. Rom. Seria Matem. Fiz., 7(4):871-886, 1955.

[188] S. Marcus. Sur un problème de F. Hausdorff concernant les fonctions symmétriques continues. Bull. Acad. Pol. Sci. (classe III), 4(4):201-205, 1956.

[189] S. Marcus. Sur un probleme de la theorie de la mesure de H. Steinhaus et $\mathrm{S}$. Ruziewicz. Bull. Acad. Plon. des Sciences, 4:197-199, 1956.

[190] S. Marcus. On anticonvex sets. Rev. Roumaine Math. Pures Appl., 13(9):1399$1401,1968$.

[191] S. Marcus. Symmetry in one dimension and symmetric continuity. Noesis, 12:98-103, 1986.

[192] S. Marcus. Symmetry in the simplest case: the real line. Comput. Math. Appl., $17: 103-115,1989$.

[193] Solomon Marcus. Fonctions convexes et fonctions internes. Bull.-Sci.-Math., $81: 66-70,1957$.

[194] J. Matoušek. A typical property of the symmetric differential quotient. Coll. Math., 57:339-343, 11989.

[195] J. Matoušek. Approximate symmetric derivative and monotonicity. Comment. Math. Univ. Carolin., 27:83-86, 1986.

[196] J. Mařik. Generalized integrals and trigonometric series. (unpublished manuscript).

[197] S. Mazurkiewicz. On the first generalized derivative (in Polish). Prace Matematyczno-Fizyczne, 28:79-85, 1917.

[198] S. Mazurkiewicz. On the relation between the existence of the second generalized derivative and the continuity of a function (in Polish). Prace MatematycznoFizyczne, 30:225-242, 1919.

[199] J. McGrotty. A theorem on complete sets. J. London Math. Soc., 37:338-340, 1962.

[200] M. R. Mehdi. On convex functions. JLMS, 39:321-326, 1964.

[201] J. C. Morgan. Point set theory. Marcel Dekker, New York, 1990.

[202] M. I. Morozov. On the question of the approximation of periodic quasi-smooth functions and functions satisfying a Lipschitz condition. Aviacion. Inst. Sergo Ord. Trudy Inst., 61:41-47, 1956.

[203] R. M. F. Moss and G. T. Roberts. A creeping lemma. Amer. Math. Monthly, $75: 649-652,1968$.

[204] S. N. Mukhopadhyay. On Schwartz differentiability, II. Rev. Roumaine Math. Pures Appl., 9:859-869, 1964.

[205] S. N. Mukhopadhyay. Some results on Schwartz derivative. Indian J. Mech. Math., 3:1-6, 1965.

[206] S. N. Mukhopadhyay. On approximate Schwartz differentiability, IV. Monatsh. Math., 70:454-460, 1966.

[207] S. N. Mukhopadhyay. On Schwartz differentiability, 1. Proc. Indian Nat. Sci. Acad. Part A, 36:525-533, 1966. 
[208] S. N. Mukhopadhyay. On differentiability. Bull. Calcutta Math. Soc, 59:181$183,1967$.

[209] S. N. Mukhopadhyay. On the regularity of the $\mathrm{P}^{n}$-integral and its application to summable trigonometric series. Pacific J. Math., 55:233-247, 1974.

[210] I. Natanson. Theory of functions of a real variable. Ungar Publishing Co., New York, 1964.

[211] C. J. Neugebauer. A theorem on derivates. Acta Sci. Math. (Szeged), 23:79-81, 1962.

[212] C. J. Neugebauer. Smoothness and differentiability in $l_{p}$. Studia Math, 25:81$91,1964$.

[213] C. J. Neugebauer. Symmetric, continuous and smooth functions. Duke Math. $J ., 31: 23-32,1964$.

[214] C. J. Neugebauer. Differentiability almost everywhere. Proc. American Math. Soc., 1205-1210, 1965.

[215] R. J. O'Malley. Approximate maxima. Fund. Math., 94:77-83, 1976.

[216] R. J. O'Malley. Baire* 1 functions. Proc. American Math. Soc., 60:187-192, 1976.

[217] D. Oppegaard Generalizations of continuity, symmetry and symmetric continuity. Thesis, North Carol. State Univ., 1993.

[218] A. Ostrowski. Zur Theorie der konvexen Funktionen. Comment. Math. Helv., 1:157-159, 1929.

[219] O. Perron. Ueber den Integralbegriff. Sitzber. Heidelberg, Akad. Wiss. Abt. A., 16:1-16, 1914.

[220] I. N. Pesin. On the measurability of symmetrically continuous functions. Teor. Funkcii Funkcional. Anal. i Prilozen, 5:99-101, 1967.

[221] G. Piranian. Two monotonic, singular, uniformmly almost smooth functions. Duke Math. J., 33:255-262, 1966.

[222] I. Pokorny. Symmetric selective derivatives. Acta Univ. Carolin. Math. Phys., 99-100, 1987.

[223] V. G. Pokrovskii, Slicings of $n$-dimensional parallelopipeds. Mat. Zametki, $33(2): 273-280,1983$.

[224] S. P. Ponomarev. On the measurability of symmetrically continuous functions. Uspehi Mat. Nauk., 20:149-150, 1965.

[225] S. P. Ponomarev. O simetričeski nèpreryvnych funkcijach. Akademiya Nauk Soyuza SSR. Matematicheskie Zametki, 1:385-390, 1967.

[226] S. P. Ponomarev. On the monogeneity of symmetrically differentiable functions. Teoret. Prikl. Mat. Vip., 2:11-14, 1967.

[227] S. P. Ponomarev. On a problem of Hausdorff. Akademiya Nauk Soyuza SSR. Matematicheskie Zametki, 14:197-200, 1973.

[228] S. P. Ponomarev. Symmetrically differentiable functions with a perfect set of points of nondifferentiability. Akademiya Nauk Soyuza SSR. Matematicheskie Zametki, 38:80-88, 1985.

[229] S. P. Ponomarev. Functions with a $D_{*}$-integrable symmetric derivative. Akademiya Nauk Soyuza SSR. Matematicheskie Zametki, 39:221-227, 1986.

[230] D. Preiss. A note on symmetrically continuous functions. Casopis Pěst. Mat., $96: 262-264,1971$. 
[231] D. Preiss and B. Thomson. A symmetric covering theorem. Real Anal. Exchange, 14:253-254, 1988/89.

[232] D. Preiss and B. Thomson. The approximate symmetric integral. Canad. J. Math., 41:508-555, 1989.

[233] H. H. Pu and H. W. Pu. Derivates for symmetric functions. Colloq. Math., 46:293-301, 1982.

[234] H.-H. Pu and H.-W. Pu. A generalization of Khintchine's theorem. Tamkang J. Math, 17:57-61, 1986.

[235] H. H. Pu and H. W. Pu. On level sets of approximate derivative and approximate symmetric derivative. Colloq. Math., 54:139-142, 1989.

[236] H. H. Pu and H. W. Pu. Pseudo-symmetric differentiation. Colloq. Math., $57: 137-143,1989$.

[237] H.-H. Pu, H.-W. Pu, and T.-H. Teng. Symmetric and approximate symmetric derivates for symmetric functions. Soochow J. Math., 5:1-7, 1979.

[238] H. W. Pu. Derivates for symmetric functions. Real Anal. Exchange, 4(2):164$166,1978 / 79$.

[239] H. W. Pu and H. H. Pu. Dini's type theorem for symmetric derivatives. Rev. Roumaine Math. Pures Appl., 18:1087-1090, 1973.

[240] H. W. Pu and H. H. Pu. Measurability of real functions having symmetric derivatives everywhere. Real Anal. Exchange, 10:214-219, 1984.

[241] H. W. Pu and H. H. Pu. A problem of Denjoy-Young-Saks theorem for symmetric derivates. Rev. Roumaine Math. Pures Appl., 33:517-518, 1988.

[242] A. Rajchman. Séries trigonométrique sommables par le procédé de Poisson (in Polish). Prace Mat.-Fiz., 30:19-88, 1919.

[243] M. Repický. An example which discerns porosity and symmetric porosity. Real Anal. Exchange, 17:416-420, 1991.

[244] M. Riesz. Uber summierbare trigonometrische Reihen. Math. Annalen, 71:54$75,1911$.

[245] A. W. Roberts and D. E. Varberg. Convex functions. Academic Press, New York, 1973.

[246] C. A. Rogers. Hausdorff Measures. Cambridge University Press, Cambridge, 1970.

[247] G. Russo. Sui numeri derivati approssimati simmetrici di une funzioune. Atti Accad. Sci. Lett. Arti Palermo Ser. (5), 4:185-196, 1984.

[248] G. Russo and S. Valenti. On the $k$-pseudo-symmetrical differentiability. Fund. Math., 94:79-83, 1981.

[249] S. Ruziewicz. Contribution à l'étude des ensembles de distances de points. Fund. Math., 7:141-143, 1925.

[250] 1. Z. Ruzsa. Locally symmetric functions. Real Anal. Exchange, 4:84-86, $1978 / 79$.

[251] S. Saks. Theory of the Integral. Monografie Matematyczne 7, Warsaw, 1937.

[252] D. Sarason. Functions of vanishing mean oscillation. Trans. American Math. Soc., 207:391-405, 1975.

[253] A.-I. Savotin. Some cases of the nondifferentiability of continuous functions that can be represented by series. Applications of functional analysis in approximation theory. Kalinin. Gos. Univ., 90-97, 1987. 
[254] P. Shanahan. A unified proof of several basic theorems of real analysis. Amer. Math. Monthly, 79:890-891, 1972.

[255] H. S. Shapiro. Monotonic singular functions of high smoothness. Michigan Math. J., 15:265-275, 1968.

[256] W. Sierpinski. Sur les fonctions convexes mesurables. Fund. Math., 1:125-128, 1920.

[257] W. Sierpinski. Sur une hypothese de S. Mazurkiewicz. Fund. Math., 11:148-150, 1928.

[258] W. Sierpinski. Sur une fonction non mesurable partout presque symétrique. Acta. Litt. Scient (Szeged), 8:1-6, 1936.

[259] V. A. Skljarenko. Certain properties of the $\mathrm{P}^{2}$-primitive. Mat. Zametki, 12:693$700,1972$.

[260] V. A. Skljarenko. On Denjoy integrable sums of everywhere convergent trigonometric series. Soviet Math. Dokl., 210:771-775, 1973.

[261] V. A. Skljarenko. Integration by parts in the SCP Burkill integral. Mat. Sb. (N.S.), 112 (154), 4(8):630-646, 1980.

[262] V. A. Skvorcov. Interrelation between general Denjoy integrals and totalization $\left(T_{2 S}\right)_{0}$. Mat. Sb. (N.S.), $52(94): 551-578,1960$.

[263] V. A. Skvorcov. Interelation between Denjoy's general integral and the totalization $\left.T_{2 S}\right)$. Dokl. Akad. Nauk SSR, 127:975-976, 1962.

[264] V. A. Skvorcov. Interrelation between the general denjoy integral and the totalization $\left.t_{2 S}\right)_{0}$. Mat. Sb. (N.S.), 52:551-578, 1962.

[265] V. A. Skvorcov. On the relation between the $D$ integral and the totalization $\left.T_{2 S}\right)$. Vestnik Moskov. Univ. Ser. I Mat. Mekh., no. 6:20-25, 1962.

[266] V. A. Skvorcov. On integrating the exact Schwarzian derivative. Mat. Sb. (N.S.), 63 (105):329-340, 1964.

[267] V. A. Skvorcov. Concerning definitions of $P^{2}$ - and SCP-integrals. Vestnik Moskov. Univ. Ser. I Mat. Mekh., 21:12-19, 1966.

[268] V. A. Skvorcov. The mutual relationship between the AP-integral of Taylor and the $P^{2}$-integral of James. Mat. Sb. (N.S.), 70 (112):380-393, 1966.

[269] V. A. Skvorcov. The connection between certain integrals. Vestnik Moskov. Univ. Ser. I Mat. Mekh., 22:68-72, 1967.

[270] V. A. Skvorcov. The Marcinkiewicz-Zygmund integral and its relation to Burkill's SCP-integral. Vestnik Moskov. Univ. Ser. I Mat. Mekh., 27:78-82, 1972.

[271] V. A. Starcev. The smoothness of functions with respect to a set. Mat. Zametki, 15:431-436, 1974.

[272] E. M. Stein. Singular Integrals and Differentiability Properties of Functions. Princeton University Press, Princeton, New Jersey, 1970.

[273] E. M. Stein. The development of square functions in the work of A. Zygmund. Conference on Harmonic Analysis in honor of Antoni Zygmund (Wadsworth), 1-30, 1983.

[274] E. M. Stein and A. Zygmund. On the differentiability of functions. Studia Math., 23:295-307, 1960.

[275] E. M. Stein and A. Zygmund. Smoothness and differentiability of functions. Ann. Univ. Sci. Budapest. Eötvös Sect. Math., III-IV:295-307, 1964. 
[276] H. Steinhaus. Problème. Fund. Math., 1: 1920.

[277] H. Steinhaus. Problème \#23. Fund. Math., p. 368, 1923.

[278] K. R. Stromberg. Universally nonmeasurable subgroups of IR. Amer. Math. Monthly, March:253-255, 1992.

[279] E. Szpilrajn. Remarque sur la dérivée symmétrique. Fund. Math., 21:226-228, 1933.

[280] S. J. Taylor. An integral of Perron's type defined with the help of trigonometric series. Quart. J. Math. Oxford Ser. (2), (2) 6:255-274, 1955.

[281] B. S. Thomson. On full covering properties. Real Anal. Exchange, 6:77-93, $1980 / 81$.

[282] B. S. Thomson. Real Functions. Lecture Notes in Mathematics 1170. SpringerVerlag, Berlin, 1985.

[283] B. S. Thomson. Derivation bases on the real line. I. II. Real Anal. Exchange, 8:67-207, 278-442, 1986 .

[284] B. S. Thomson. Some symmetric covering lemmas. Real Anal. Exchange, $15: 346-383,1989$.

[285] B. S. Thomson. An analogue of Charzyński's theorem. Real Anal. Exchange, $15: 743-753,1989 / 90$.

[286] B. S. Thomson. Derivates of interval functions. Mem. Amer. Math. Soc., 93, no. 452,96 pp., 1991.

[287] B. S. Thomson. Symmetric variation. Real Anal. Exchange, 17:409-415, 1991/92.

[288] B. S. Thomson. The range of symmetric derivatives. Real Anal. Exchange, (to appear) 1993.

[289] A. F. Timan. Quasi-smooth functions. Uspehi Matem. Nauk (N.S.), 5:128-130, 1950.

[290] A. F. Timan. On quasi-smooth functions. Izvestiya Akad. Nauk-SSSR Ser. Mat., 15:243-254, 1951.

[291] L. Tonelli. Su una proposizione fondamentale dell'analisi matematica. Rend $R$. Accad. die Lincei, 23:161-165, 1936.

[292] T. C. Tran. Symmetric functions whose set of points of discontinuity is uncountable. Real Anal. Exchange, 12:496-508, 1986/87.

[293] J. Uher. Symmetrically differentiable functions are differentiable almost everywhere. Real Anal. Exchange, 8:253-260, 1982/83.

[294] J. Uher. Symmetric continuity implies continuity. Trans. American Math. Soc., 293:421-429, 1986.

[295] J. Uher. Symmetric continuity implies continuity. Real Anal. Exchange, 13:35$38,1987 / 88$.

[296] D. C. Ullrich. Differentiation of Zygmund functions. Proc. Amer. Math. Soc., 117:195-197, 1993.

[297] S. Valenti. Sur la derivation $k$-pseudo-symetrique des fonctions numeriques. Fund. Math., 74:147-152, 1972.

[298] R. W. Vallin. Shell porous, porous, totally porous, hyperporous sets. Real Anal. Exchange, 18:294-320, 1992/93.

[299] S. Verblunsky. The generalized fourth derivative. J. London Math. Soc., 6:82$84,1931$. 
[300] S. Verblunsky. On the theory of trigonometrical series (VI). Proc. London Math. Soc., 38:284-326, 1934.

[301] S. Verblunsky. On the theory of trigonometrical series (VII). Fund. Math., 23:192-236, 1934.

[302] T. Viola. Sur la détermination d'une fonction discontinue par sa dérivée unilatérale. Ann. École Norm., (3) 50:72-125, 1933.

[303] E. Wajch. On symmetric derivatives of functions of the first class of Baire. Demonstratio Math., 19:189-195, 1986.

[304] C. E. Weil. Monotonicity, convexity and symmetric derivatives. Trans. American Math. Sac., 222:225-237, 1976.

[305] C. E. Weil. The Peano derivative: what's known and what isn't. Real Anal. Exchange, 9:354-365, 1983/84.

[306] M. Weiss. On symmetric derivatives in $L^{p}$. Studia Math., 24:89-100, 1964.

[307] M. Weiss and A. Zygmund. A note on smooth functions. Konin. Nederlandse Akad. van Wetenschappen Proc., 62:52-58, 1959.

[308] W. H. Young and G. C. Young. On the reduction of sets of intervals. Proc. London Math. Soc. (2), 14:111-130, 1915.

[309] Z. Zahorski. Sur la première derivée. Trans. American Math. Soc., 69:1-54, 1950.

[310] L. Zajíček. Porosity and $\sigma$-porosity. Real Anal. Exchange, 13:314-350, 1987/88.

[311] L. Zajičck. A note on the symmetric and ordinary derivative. (manuscript), 1991.

[312] A. Zygmund. Smooth functions. Duke Math. J., 12:47-76, 1945.

[313] A. Zygmund. Trigonometric series. Cambridge University Press, London, 1968.

[314] A. Zygmund. Selected Papers of Antoni Zygmund, edited by A. Hulanicki, P. Wojtaszczyk, and W. Zelazko. Kluwer Academic Publ. (London), 1989. 